\title{
Bounded Geometry and $p$-Harmonic Functions Under Uniformization and Hyperbolization
}

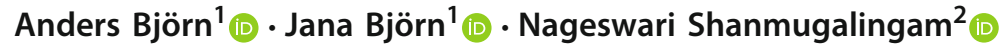

Received: 27 February 2020 / Published online: 30 July 2020

(c) The Author(s) 2020

\begin{abstract}
The uniformization and hyperbolization transformations formulated by Bonk et al. in “Uniformizing Gromov Hyperbolic Spaces”, Astérisque, vol 270 (2001), dealt with geometric properties of metric spaces. In this paper we consider metric measure spaces and construct a parallel transformation of measures under the uniformization and hyperbolization procedures. We show that if a locally compact roughly starlike Gromov hyperbolic space is equipped with a measure that is uniformly locally doubling and supports a uniformly local $p$-Poincaré inequality, then the transformed measure is globally doubling and supports a global $p$-Poincaré inequality on the corresponding uniformized space. In the opposite direction, we show that such global properties on bounded locally compact uniform spaces yield similar uniformly local properties for the transformed measures on the corresponding hyperbolized spaces. We use the above results on uniformization of measures to characterize when a Gromov hyperbolic space, equipped with a uniformly locally doubling measure supporting a uniformly local $p$-Poincaré inequality, carries nonconstant globally defined $p$-harmonic functions with finite $p$-energy. We also study some geometric properties of Gromov hyperbolic and uniform spaces. While the Cartesian product of two Gromov hyperbolic spaces need not be Gromov hyperbolic, we construct an indirect product of such spaces that does result in a Gromov hyperbolic space. This is done by first showing that the Cartesian product of two bounded uniform domains is a uniform domain.
\end{abstract}

Keywords Bounded geometry · Doubling measure $\cdot$ Finite-energy Liouville theorem · Gromov hyperbolic space $\cdot$ Hyperbolization $\cdot$ Metric space $\cdot p$-harmonic function · Poincaré inequality · Quasihyperbolic metric · Uniform space · Uniformization

Mathematics Subject Classification Primary 53C23 - Secondary 30F10, 30L10, 30L99, 31E05

Dedicated to Pekka Koskela and the late Juha Heinonen on their 59th birthdays.

Extended author information available on the last page of the article 


\section{Introduction}

Studies of metric space geometry usually consider two types of synthetic (i.e. axiomatic) negative curvature conditions: Alexandrov curvature (known as CAT $(-1)$ spaces) and Gromov hyperbolicity. While the Alexandrov condition governs both small and large scale behavior of triangles, the Gromov hyperbolicity governs only the large scale behavior. As such, the Gromov hyperbolicity was eminently suited to the study of hyperbolic groups, see e.g. Gromov [24], Coornaert-Delzant-Papadopoulos [20] and Ghys-de la Harpe [23], while Bridson-Haefliger [17] gives an excellent overview of both notions of curvature.

Since the ground-breaking work of Gromov [24], the notion of Gromov hyperbolicity has found applications in other parts of metric space analysis as well. In [14, Theorem 1.1], Bonk, Heinonen and Koskela gave a link between quasiisometry classes of locally compact roughly starlike Gromov hyperbolic spaces and quasisimilarity classes of locally compact bounded uniform spaces. In Buyalo-Schroeder [19] it was shown that every complete bounded doubling metric space is the visual boundary of a Gromov hyperbolic space, see also Bonk-Saksman [15].

While none of the above mentioned studies, involving Gromov hyperbolic spaces and uniform domains, considered how measures transform on such spaces (see also e.g. Buckley-Herron-Xie [18] and Herron-Shanmugalingam-Xie [31]), analytic studies on metric spaces require measures as well. Although [15] does consider function spaces on certain Gromov hyperbolic spaces, called hyperbolic fillings, these function spaces are associated with just the counting measure on the vertices of such hyperbolic fillings and so do not lend themselves to more general Gromov hyperbolic spaces. Similar studies were undertaken in Bonk-Saksman-Soto [16] and Björn-Björn-GillShanmugalingam [8].

In this paper we seek to remedy this gap in the literature on analysis in Gromov hyperbolic spaces. Thus the primary focus of this paper is to construct transformations of measures under the uniformization and hyperbolization procedures, and to demonstrate how analytic properties of the measure are preserved by them. This does not seem to have been considered elsewhere. The analytic properties of interest here are the doubling property and the Poincaré inequality, assumed either globally on the uniform spaces, or uniformly locally (i.e. for balls up to some fixed radius) on the Gromov hyperbolic spaces. As trees are the quintessential models of Gromov hyperbolic spaces, the results in this paper are motivated in part by the results in [8].

The following is our main result, combining Theorems 4.9 and 6.2. Here, $z_{0} \in X$ is a fixed uniformization center and $\varepsilon_{0}(\delta)$ is as in Bonk-Heinonen-Koskela [14], see later sections for relevant definitions.

Theorem 1.1 Assume that $(X, d)$ is a locally compact roughly starlike Gromov $\delta$ hyperbolic space equipped with a measure $\mu$ which is doubling on $X$ for balls of radii at most $R_{0}$, with a doubling constant $C_{d}$. Let $X_{\varepsilon}=\left(X, d_{\varepsilon}\right)$ be the uniformization of $X$ given for $0<\varepsilon \leq \varepsilon_{0}(\delta)$ by

$$
d_{\varepsilon}(x, y)=\inf _{\gamma} \int_{\gamma} e^{-\varepsilon d\left(\cdot, z_{0}\right)} \mathrm{d} s,
$$


with the infimum taken over all rectifiable curves $\gamma$ in $X$ joining $x$ to $y$. Also let

$$
\beta>\frac{17 \log C_{d}}{3 R_{0}} \text { and } \mathrm{d} \mu_{\beta}=e^{-\beta d\left(\cdot, z_{0}\right)} \mathrm{d} \mu .
$$

Then the following are true:

(a) $\mu_{\beta}$ is globally doubling both on $X_{\varepsilon}$ and its completion $\bar{X}_{\varepsilon}$.

(b) If $\mu$ supports a $p$-Poincaré inequality for balls of radii at most $R_{0}$, then $\mu_{\beta}$ supports a global p-Poincaré inequality both on $X_{\varepsilon}$ and $\bar{X}_{\varepsilon}$.

Along the way, we also show that if the assumptions hold with some value of $R_{0}$ then they hold for any value of $R_{0}$ at the cost of enlarging $C_{d}$, see Proposition 3.2 and Theorem 5.3.

We also obtain the following corresponding result for the hyperbolization procedure, see Propositions 7.3 and 7.4.

Theorem 1.2 Let $(\Omega, d)$ be a locally compact bounded uniform space, equipped with a globally doubling measure $\mu$. Let $k$ be the quasihyperbolic metric on $\Omega$, given by

$$
k(x, y)=\inf _{\gamma} \int_{\gamma} \frac{\mathrm{d} s}{d_{\Omega}(\cdot)},
$$

where $d_{\Omega}(x)=\operatorname{dist}(x, \partial \Omega)$ and the infimum is taken over all rectifiable curves $\gamma$ in $\Omega$ connecting $x$ to $y$. For $\alpha>0$ we equip the corresponding Gromov hyperbolic space $(\Omega, k)$ with the measure $\mu^{\alpha}$ given by $\mathrm{d} \mu^{\alpha}=d_{\Omega}(\cdot)^{-\alpha} \mathrm{d} \mu$. Let $R_{0}>0$.

Then the following are true:

(a) $\mu^{\alpha}$ is doubling on $(\Omega, k)$ for balls of radii at most $R_{0}$.

(b) If $\mu$ supports a global p-Poincaré inequality, then $\mu^{\alpha}$ supports a p-Poincaré inequality for balls of radii at most $R_{0}$.

We use Theorem 1.1 to study potential theory on locally compact roughly starlike Gromov hyperbolic spaces, equipped with a locally uniformly doubling measure supporting a uniformly local Poincaré inequality. In particular, we characterize when the finite-energy Liouville theorem holds on such spaces, i.e. when there exist no nonconstant globally defined $p$-harmonic functions with finite $p$-energy. The characterization is given in terms of the nonexistence of two disjoint compact sets of positive $p$-capacity in the boundary of the uniformized space, see Theorem 10.5. This characterization complements our results in [12].

As already mentioned, an in-depth study of locally compact roughly starlike Gromov hyperbolic spaces, as well as links between them and bounded locally compact uniform domains, was undertaken in the seminal work Bonk-Heinonen-Koskela [14]. They showed [14, the discussion before Proposition 4.5] that the operations of uniformization and hyperbolization are mutually opposite:

- A uniformization followed by a hyperbolization takes a given locally compact roughly starlike Gromov hyperbolic space $X$ to a roughly starlike Gromov hyperbolic space which is biLipschitz equivalent to $X$, see [14, Proposition 4.37]. (Note that in [14] "quasiisometric" means biLipschitz.) 
- A hyperbolization of a bounded locally compact uniform space $\Omega$, followed by a uniformization, returns a bounded uniform space which is quasisimilar to $\Omega$, see [14, Proposition 4.28].

Here, a homeomorphism $\Phi: X \rightarrow Y$ between two noncomplete metric spaces is quasisimilar if it is $C_{x}$-biLipschitz on every ball $B\left(x, c_{0} \operatorname{dist}(x, \partial X)\right)$, for some $0<c_{0}<1$ independent of $x$, and there exists a homeomorphism $\eta:[0, \infty) \rightarrow[0, \infty)$ such that for each distinct triple of points $x, y, z \in X$,

$$
\frac{d_{Y}(\Phi(x), \Phi(y))}{d_{Y}(\Phi(x), \Phi(z))} \leq \eta\left(\frac{d_{X}(x, y)}{d_{X}(x, z)}\right)
$$

It was also shown in [14, Theorem 4.36] that two roughly starlike Gromov hyperbolic spaces are biLipschitz equivalent if and only if any two of their uniformizations are quasisimilar.

We continue the study of Gromov hyperbolic spaces in this spirit by considering pairs of Gromov hyperbolic spaces in Sect. 8. Note that the Cartesian product of two Gromov hyperbolic spaces need not be Gromov hyperbolic, as demonstrated by $\mathbf{R} \times \mathbf{R}$, which is not a Gromov hyperbolic space even though $\mathbf{R}$ is. On the other hand, in Sect. 8 we obtain the following result, see Proposition 8.3 for a more precise result.

Proposition 1.3 Let $(\Omega, d)$ and $\left(\Omega^{\prime}, d^{\prime}\right)$ be two bounded uniform spaces. Then $\Omega \times \Omega^{\prime}$ is a bounded uniform space with respect to the metric

$$
\tilde{d}\left(\left(x, x^{\prime}\right),\left(y, y^{\prime}\right)\right)=d(x, y)+d^{\prime}\left(x^{\prime}, y^{\prime}\right) .
$$

We use this, together with the results of [14], to construct an indirect product $X \times{ }_{\varepsilon} Y$ of two Gromov hyperbolic spaces which is also Gromov hyperbolic, see Sect. 8. In this section we also study properties of such product hyperbolic spaces. For a fixed Gromov $\delta$-hyperbolic space $X$, there is a whole family of uniformizations $X_{\varepsilon}$, one for each $0<\varepsilon \leq \varepsilon_{0}(\delta)$. As mentioned above, $X_{\varepsilon}$ is quasisimilar to $X_{\varepsilon^{\prime}}$ when $0<\varepsilon, \varepsilon^{\prime} \leq \varepsilon_{0}(\delta)$.

On the other hand, we show in Proposition 8.5 that the canonical identity mapping between $X \times{ }_{\varepsilon} Y$ and $X \times{ }_{\varepsilon^{\prime}} Y$ is never biLipschitz if $\varepsilon \neq \varepsilon^{\prime}$, and it is even possible that the two indirect products are not even quasiisometric. Here, a map $\Phi: Z \rightarrow W$ is a quasiisometry (also called, perhaps more accurately, rough quasiisometry as in [14] and [8]) if there are $C>0$ and $L \geq 1$ such that the $C$-neighborhood of $\Phi(Z)$ contains $W$ and for all $z, z^{\prime} \in Z$,

$$
\frac{d\left(z, z^{\prime}\right)}{L}-C \leq d\left(\Phi(z), \Phi\left(z^{\prime}\right)\right) \leq L d\left(z, z^{\prime}\right)+C
$$

It is not difficult to show that visual boundaries of quasiisometric locally compact roughly starlike Gromov hyperbolic spaces are quasisymmetric, see e.g. BridsonHaefliger [17, Theorem 3.22]. We take advantage of this to show the quasiisometric nonequivalence of two indirect products of the hyperbolic disk and $\mathbf{R}$, see Example 8.7. 
The broad organization of the paper is as follows. Background definitions and preliminary results are given in Sects. 2 and 3, while the definition of Poincaré inequalities is given in Sect. 5. The main aims in Sects. 4 and 6 are to deduce parts (a) and (b), respectively, of Theorem 1.1. The dual transformation of hyperbolization, via the quasihyperbolic metric (1.1), is discussed in Sect. 7, where also Theorem 1.2 is shown. The above sections fulfill the main goal of this paper, and form a basis for comparing the potential theories on Gromov hyperbolic spaces and on uniform spaces.

The remaining sections are devoted to applications of the results obtained in the preceding sections. In Sect. 8 we construct and study the indirect product, providing a family of new Gromov hyperbolic spaces from a pair of Gromov hyperbolic spaces. The subsequent sections are devoted to the impact of uniformization and hyperbolization procedures on nonlinear potential theory. In Sect. 9 we discuss Newton-Sobolev spaces and $p$-harmonic functions, and then in Sect. 10 we show that under certain natural conditions, the class of $p$-harmonic functions is preserved under the uniformization and hyperbolization procedures. In this final section, we also characterize which Gromov hyperbolic spaces with bounded geometry support the finite-energy Liouville theorem for $p$-harmonic functions.

In the beginning of each section, we list the standing assumptions for that section in italicized text; in Sects. 2 and 4 these assumptions are given a little later.

\section{Gromov Hyperbolic Spaces}

A curve is a continuous mapping from an interval. Unless stated otherwise, we will only consider curves which are defined on compact intervals. We denote the length of a curve $\gamma$ by $l_{\gamma}=l(\gamma)$, and a curve is rectifiable if it has finite length. Rectifiable curves can be parametrized by arc length $\mathrm{d} s$.

A metric space $X=(X, d)$ is $L$-quasiconvex if for each $x, y \in X$ there is a curve $\gamma$ with end points $x$ and $y$ and length $l_{\gamma} \leq L d(x, y) . X$ is a geodesic space if it is 1-quasiconvex, and $\gamma$ is then a geodesic from $x$ to $y$. We will consider a related metric, called the inner metric, given by

$$
d_{\text {in }}(x, y):=\inf _{\gamma} l_{\gamma} \text { for all } x, y \in X
$$

where the infimum is taken over all curves $\gamma$ from $x$ to $y$. If $(X, d)$ is quasiconvex, then $d$ and $d_{\text {in }}$ are biLipschitz equivalent metrics on $X$. The space $X$ is a length space if $d_{\text {in }}(x, y)=d(x, y)$ for all $x, y \in X$. By Lemma 4.43 in [5], arc length is the same with respect to $d$ and $d_{\text {in }}$, and thus $\left(X, d_{\text {in }}\right)$ is a length space. A metric space is proper if all closed bounded sets are compact. A proper length space is necessarily a geodesic space, by Ascoli's theorem or the Hopf-Rinow theorem below. To avoid pathological situations, all metric spaces in this paper are assumed to contain at least two points.

We denote balls in $X$ by $B(x, r)=\{y \in X: d(y, x)<r\}$ and the scaled concentric ball by $\lambda B(x, r)=B(x, \lambda r)$. In metric spaces it can happen that balls with different centers and/or radii denote the same set. We will however adopt the convention that a ball comes with a predetermined center and radius. Similarly, when we say that $x \in \gamma$ 
we mean that $x=\gamma(t)$ for some $t$. If $\gamma$ is noninjective, this $t$ may not be unique, but we are always implicitly referring to a specific such $t$.

Theorem 2.1 (Hopf-Rinow theorem) If X is a complete locally compact length space, then it is proper and geodesic.

This version is a generalization of the original theorem, see e.g. Gromov [25, p. 9] for a proof.

Definition 2.2 A complete unbounded geodesic metric space $X$ is Gromov hyperbolic if there is a hyperbolicity constant $\delta \geq 0$ such that whenever $[x, y],[y, z]$ and $[z, x]$ are geodesics in $X$, every point $w \in[x, y]$ lies within a distance $\delta$ of $[y, z] \cup[z, x]$.

The ideal Gromov hyperbolic space is a metric tree, which is Gromov hyperbolic with $\delta=0$. A metric tree is a tree where each edge is considered to be a geodesic of unit length.

Definition 2.3 An unbounded metric space $X$ is roughly starlike if there are some $z_{0} \in X$ and $M>0$ such that whenever $x \in X$ there is a geodesic ray $\gamma$ in $X$, starting from $z_{0}$, such that $\operatorname{dist}(x, \gamma) \leq M$. A geodesic ray is a curve $\gamma:[0, \infty) \rightarrow X$ with infinite length such that $\left.\gamma\right|_{[0, t]}$ is a geodesic for each $t>0$.

If $X$ is a roughly starlike Gromov hyperbolic space, then the roughly starlike condition holds for every choice of $z_{0}$, although $M$ may change.

Definition 2.4 A nonempty open set $\Omega \varsubsetneqq X$ in a metric space $X$ is an A-uniform domain, with $A \geq 1$, if for every pair $x, y \in \Omega$ there is a rectifiable arc length parametrized curve $\gamma:\left[0, l_{\gamma}\right] \rightarrow \Omega$ with $\gamma(0)=x$ and $\gamma\left(l_{\gamma}\right)=y$ such that $l_{\gamma} \leq A d(x, y)$ and

$$
d_{\Omega}(\gamma(t)) \geq \frac{1}{A} \min \left\{t, l_{\gamma}-t\right\} \text { for } 0 \leq t \leq l_{\gamma}
$$

where

$$
d_{\Omega}(z)=\operatorname{dist}(z, X \backslash \Omega), \quad z \in \Omega
$$

The curve $\gamma$ is said to be an A-uniform curve. A noncomplete metric space $(\Omega, d)$ is $A$-uniform if it is an $A$-uniform domain in its completion.

A ball $B(x, r)$ in a uniform space $\Omega$ is a subWhitney ball if $r \leq c_{0} d_{\Omega}(x)$, where $0<c_{0}<1$ is a predetermined constant. We will primarily use $c_{0}=\frac{1}{2}$.

The completion of a locally compact uniform space is always proper, by Proposition 2.20 in Bonk-Heinonen-Koskela [14]. Unlike the definition used in [14], we do not require uniform spaces to be locally compact.

It follows directly from the definition that an $A$-uniform space is $A$-quasiconvex. One might ask if the uniformity assumption in Proposition 2.20 in [14] can be replaced by a quasiconvexity assumption, i.e. if the completion of a locally compact quasiconvex space is always proper, however the following example shows that this can fail even if the original space is geodesic. Thus the uniformity assumption in Proposition 2.20 in [14] is really crucial. 


\section{Example 2.5 Let}

$$
X=\left\{\left\{x_{j}\right\}_{j=1}^{\infty}: \sum_{j=1}^{\infty}\left|x_{j}\right| \leq 1,0<x_{1} \leq 1 \text {, and } x_{n}=0 \text { if } x_{1}>\frac{1}{n}, n=2,3, \ldots\right\}
$$

equipped with the $\ell^{1}$-metric. Then $X$ is a bounded locally compact geodesic space which is not totally bounded, and thus has a nonproper completion.

We assume from now on that $X$ is a locally compact roughly starlike Gromov $\delta$ hyperbolic space. We also fix a point $z_{0} \in X$ and let $M$ be the constant in the roughly starlike condition with respect to $z_{0}$.

By the Hopf-Rinow Theorem 2.1, $X$ is proper. The point $z_{0}$ will serve as a center for the uniformization $X_{\varepsilon}$ of $X$. Following Bonk-Heinonen-Koskela [14], we define

$$
(x \mid y)_{z_{0}}:=\frac{1}{2}\left[d\left(x, z_{0}\right)+d\left(y, z_{0}\right)-d(x, y)\right], \quad x, y \in X
$$

and, for a fixed $\varepsilon>0$, the uniformized metric $d_{\varepsilon}$ on $X$ as

$$
d_{\varepsilon}(x, y)=\inf _{\gamma} \int_{\gamma} \rho_{\varepsilon} \mathrm{d} s, \quad \text { where } \rho_{\varepsilon}(x)=e^{-\varepsilon d\left(x, z_{0}\right)}
$$

and the infimum is taken over all rectifiable curves $\gamma$ in $X$ joining $x$ to $y$. Note that if $\gamma$ is a compact curve in $X$, then $\rho_{\varepsilon}$ is bounded from above and away from 0 on $\gamma$, and in particular $\gamma$ is rectifiable with respect to $d_{\varepsilon}$ if and only if it is rectifiable with respect to $d$.

The set $X$, equipped with the metric $d_{\varepsilon}$, is denoted by $X_{\varepsilon}$. We let $\bar{X}_{\varepsilon}$ be the completion of $X_{\varepsilon}$, and let $\partial_{\varepsilon} X=\bar{X}_{\varepsilon} \backslash X_{\varepsilon}$. When writing e.g. $B_{\varepsilon}, \operatorname{diam}_{\varepsilon}$ and dist $\operatorname{din}_{\varepsilon}$ the indicates that these notions are taken with respect to $\left(\bar{X}_{\varepsilon}, d_{\varepsilon}\right)$. The length of the curve $\gamma$ with respect to $d_{\varepsilon}$ is denoted by $l_{\varepsilon}(\gamma)$, and arc length $\mathrm{d} s_{\varepsilon}$ with respect to $d_{\varepsilon}$ satisfies

$$
\mathrm{d} s_{\varepsilon}=\rho_{\varepsilon} \mathrm{d} s
$$

It follows that $X_{\varepsilon}$ is a length space, and thus also $\bar{X}_{\varepsilon}$ is a length space. By a direct calculation (or $[14,(4.3)]$ ), $\operatorname{diam}_{\varepsilon} \bar{X}_{\varepsilon}=\operatorname{diam}_{\varepsilon} X_{\varepsilon} \leq 2 / \varepsilon$. Note that as a set, $\partial_{\varepsilon} X$ is independent of $\varepsilon$ and depends only on the Gromov hyperbolic structure of $X$, see e.g. [14, Sect. 3]. The notation adopted in [14] is $\partial_{G} X$.

The following important theorem is due to Bonk-Heinonen-Koskela [14].

Theorem 2.6 There is a constant $\varepsilon_{0}=\varepsilon_{0}(\delta)>0$ only depending on $\delta$ such that if $0<\varepsilon \leq \varepsilon_{0}(\delta)$, then $X_{\varepsilon}$ is an A-uniform space for some A depending only on $\delta$, and $\bar{X}_{\varepsilon}$ is a compact geodesic space.

If $\delta=0$, then $\varepsilon_{0}(0)$ can be chosen arbitrarily large.

In the proof below we recall the relevant references from [14] and specify the dependence on $\delta$. 
Proof By Proposition 4.5 in [14] there is $\varepsilon_{0}(\delta)>0$ such that if $0<\varepsilon \leq \varepsilon_{0}(\delta)$, then $X_{\varepsilon}$ is an $A$-uniform space for some $A$ depending only on $\delta$. As $X_{\varepsilon}$ is bounded, it follows from Proposition 2.20 in [14] that $\bar{X}_{\varepsilon}$ is a compact length space, which by Ascoli's theorem or the Hopf-Rinow Theorem 2.1 is geodesic.

The bound $\varepsilon_{0}(\delta)$ in Proposition 4.5 in [14] is only needed for the Gehring-Hayman lemma to be true, see [14, Theorem 5.1]. If $\delta=0$, then any curve from $x$ to $y$ contains the unique geodesic $[x, y]$ as a subcurve. From this the Gehring-Hayman lemma follows directly without any bound on $\varepsilon$. Note that in this case it also follows that a curve in $X=X_{\varepsilon}$ is simultaneously a geodesic with respect to $d$ and $d_{\varepsilon}$.

We recall, for further reference, the following key estimates from [14].

Lemma 2.7 ([14, Lemma 4.10]) There exists a constant $C(\delta) \geq 1$ such that for every $0<\varepsilon \leq \varepsilon_{0}=\varepsilon_{0}(\delta)$ and all $x, y \in X$,

$$
\frac{1}{C(\delta)} d_{\varepsilon}(x, y) \leq \frac{\exp \left(-\varepsilon(x \mid y)_{z_{0}}\right)}{\varepsilon} \min \{1, \varepsilon d(x, y)\} \leq C(\delta) d_{\varepsilon}(x, y) .
$$

Lemma 2.8 ([14, Lemma 4.16]) Let $\varepsilon>0$. If $x \in X$, then

$$
\frac{e^{-\varepsilon d\left(x, z_{0}\right)}}{e \varepsilon} \leq \operatorname{dist}_{\varepsilon}\left(x, \partial_{\varepsilon} X\right)=: d_{\varepsilon}(x) \leq C_{0} \frac{e^{-\varepsilon d\left(x, z_{0}\right)}}{\varepsilon},
$$

where $C_{0}=2 e^{\varepsilon M}-1$. In particular, $\varepsilon d_{\varepsilon}(x) \simeq \rho_{\varepsilon}(x)$, and $x \rightarrow \partial_{\varepsilon} X$ with respect to $d_{\varepsilon}$ if and only if $d\left(x, z_{0}\right) \rightarrow \infty$.

Note that one may choose $C_{0}=2 e^{\varepsilon_{0} M}-1$ for it to be independent of $\varepsilon$, provided that $0<\varepsilon \leq \varepsilon_{0}$.

Corollary 2.9 Assume that $0<\varepsilon \leq \varepsilon_{0}(\delta)$. Let $x, y \in X$. If $\varepsilon d(x, y) \geq 1$ then

$$
\exp (\varepsilon d(x, y)) \simeq \frac{d_{\varepsilon}(x, y)^{2}}{d_{\varepsilon}(x) d_{\varepsilon}(y)}
$$

where the comparison constants depend only on $\delta, M$ and $\varepsilon_{0}$.

Proof Since $\varepsilon d(x, y) \geq 1,(2.2)$ can be written as

$$
\exp \left(-2 \varepsilon(x \mid y)_{z_{0}}\right) \simeq\left(\varepsilon d_{\varepsilon}(x, y)\right)^{2}
$$

where the comparison constants depend only on $\delta$. Moreover, (2.3) gives

$$
\exp \left(-\varepsilon d\left(x, z_{0}\right)\right) \simeq \varepsilon d_{\varepsilon}(x) \text { and } \exp \left(-\varepsilon d\left(y, z_{0}\right)\right) \simeq \varepsilon d_{\varepsilon}(y)
$$

with comparison constants depending only on $M$ and $\varepsilon_{0}$. Dividing (2.4) by the last two formulas, and using the definition of $(x \mid y)_{z_{0}}$ concludes the proof.

We now wish to show that subWhitney balls in the uniformization $X_{\varepsilon}$ are contained in balls of a fixed radius with respect to the Gromov hyperbolic metric $d$ of $X$. 
Theorem 2.10 For all $0<\varepsilon \leq \varepsilon_{0}(\delta), x \in X$ and $0<r \leq \frac{1}{2} d_{\varepsilon}(x)$, we have

$$
B\left(x, \frac{C_{1} r}{\rho_{\varepsilon}(x)}\right) \subset B_{\varepsilon}(x, r) \subset B\left(x, \frac{C_{2} r}{\rho_{\varepsilon}(x)}\right),
$$

where $C_{1}=e^{-(1+\varepsilon M)}$ and $C_{2}=2 e\left(2 e^{\varepsilon M}-1\right)$. If $d_{\varepsilon}(x, y)<C_{1} d_{\varepsilon}(x) / 2 C_{2}$, then

$$
\frac{\rho_{\varepsilon}(x)}{C_{2}} d(x, y)<d_{\varepsilon}(x, y) \leq e^{1 / e} \rho_{\varepsilon}(x) d(x, y) .
$$

Remark 2.11 As in Lemma 2.8, the constants $C_{1}$ and $C_{2}$ obtained for $\varepsilon_{0}$ will do for $\varepsilon<\varepsilon_{0}$ as well. The proof also shows that the condition $0<r \leq \frac{1}{2} d_{\varepsilon}(x)$ can be replaced by $0<r \leq c_{0} d_{\varepsilon}(x)$ for any fixed $0<c_{0}<1$, but then $C_{1}$ and $C_{2}$ also depend on $c_{0}$ and get progressively worse as $c_{0}$ approaches 1 .

Proof Assume that $y \in B\left(x, C_{1} r / \rho_{\varepsilon}(x)\right)$ and let $\gamma$ be a $d$-geodesic from $x$ to $y$. The assumption $r \leq \frac{1}{2} d_{\varepsilon}(x)$ and (2.3) then imply that for all $z \in \gamma$,

$$
d(x, z) \leq d(x, y)<\frac{C_{1} r}{\rho_{\varepsilon}(x)} \leq \frac{C_{1} d_{\varepsilon}(x)}{2 \rho_{\varepsilon}(x)} \leq \frac{C_{1}\left(2 e^{\varepsilon M}-1\right)}{2 \varepsilon}<\frac{C_{1} e^{\varepsilon M}}{\varepsilon}=\frac{1}{\varepsilon e} .
$$

The triangle inequality then yields $d\left(z, z_{0}\right) \geq d\left(x, z_{0}\right)-d(x, z) \geq d\left(x, z_{0}\right)-1 / \varepsilon e$ and hence

$$
\rho_{\varepsilon}(z)=e^{-\varepsilon d\left(z, z_{0}\right)} \leq e^{1 / e} \rho_{\varepsilon}(x)
$$

From this and (2.5) it readily follows that

$$
d_{\varepsilon}(x, y) \leq \int_{\gamma} \rho_{\varepsilon} \mathrm{d} s \leq e^{1 / e} \rho_{\varepsilon}(x) d(x, y)<C_{1} e^{1 / e} r<r .
$$

To see the other inclusion, assume that $d_{\varepsilon}(x, y)<r \leq \frac{1}{2} d_{\varepsilon}(x)$ and let $\gamma_{\varepsilon}$ be a geodesic curve in $\bar{X}_{\varepsilon}$ connecting $x$ to $y$. Then for all $z \in \gamma_{\varepsilon}$, we have by the triangle inequality that

$$
d_{\varepsilon}(z) \geq d_{\varepsilon}(x)-d_{\varepsilon}(x, z) \geq d_{\varepsilon}(x)-d_{\varepsilon}(x, y)>\frac{1}{2} d_{\varepsilon}(x),
$$

in particular $\gamma_{\varepsilon} \subset X_{\varepsilon}$. It now follows from (2.3) that

$$
\rho_{\varepsilon}(z) \geq \frac{\varepsilon d_{\varepsilon}(z)}{2 e^{\varepsilon M}-1}>\frac{\varepsilon d_{\varepsilon}(x)}{2\left(2 e^{\varepsilon M}-1\right)} \geq \frac{\rho_{\varepsilon}(x)}{C_{2}},
$$

where $C_{2}$ is as in the statement of the theorem. This implies that

$$
r>d_{\varepsilon}(x, y)=\int_{\gamma_{\varepsilon}} \rho_{\varepsilon} \mathrm{d} s>\frac{\rho_{\varepsilon}(x)}{C_{2}} d(x, y),
$$


and hence $B_{\varepsilon}(x, r) \subset B\left(x, C_{2} r / \rho_{\varepsilon}(x)\right)$.

Finally, if $d_{\varepsilon}(x, y)<C_{1} d_{\varepsilon}(x) / 2 C_{2}$, then from the last inclusion above we see that $y \in B\left(x, C_{1} s / \rho_{\varepsilon}(x)\right)$ with $s=\frac{1}{2} d_{\varepsilon}(x)$. Therefore we can apply (2.6) and (2.7) to obtain the last claim of the lemma.

In this paper, the letter $C$ will denote various positive constants whose values may change even within a line. We write $Y \lesssim Z$ if there is an implicit constant $C>0$ such that $Y \leq C Z$, and analogously $Y \gtrsim Z$ if $Z \lesssim Y$. We also use the notation $Y \simeq Z$ to mean $Y \lesssim Z \lesssim Y$. We will point out how the comparison constants depend on various other constants related to the metric measure spaces under study.

\section{Doubling Property}

In the rest of this paper, we will continue to assume that $X$ is a locally compact roughly starlike Gromov hyperbolic space. For general definitions and some results, we will assume that $Y$ is a metric space equipped with a Borel measure $v$.

Just as for $X$, we will denote the metric on $Y$ by $d$, and balls in $Y$ by $B(x, r)$, but it should always be clear from the context in which space these concepts are taken.

Definition 3.1 A Borel measure $v$, defined on a metric space $Y$, is globally doubling if

$$
0<v(B(x, 2 r)) \leq C_{d} v(B(x, r))<\infty
$$

whenever $x \in Y$ and $r>0$. If this holds only for balls of radii $\leq R_{0}$, then we say that $v$ is doubling for balls of radii at most $R_{0}$, and also that $v$ is uniformly locally doubling.

The following result shows that the last condition is independent of $R_{0}$, provided that $Y$ is quasiconvex. Without assuming quasiconvexity this is not true as shown by Example 3.3 below.

Proposition 3.2 Assume that $Y$ is L-quasiconvex and that $v$ is doubling on $Y$ for balls of radii at most $R_{0}$, with a doubling constant $C_{d}$. Let $R_{1}>0$. Then $v$ is doubling on $Y$ for balls of radii at most $R_{1}$ with a doubling constant depending only on $R_{1} / R_{0}, L$ and $C_{d}$.

Example 3.3 Let $X=([0, \infty) \times\{0,1\}) \cup(\{0\} \times[0,1])$ equipped with the Euclidean distance and the measure $\mathrm{d} \mu=w d \mathcal{L}^{1}$, where $\mathcal{L}^{1}$ is the Lebesgue measure and

$$
w(x, y)= \begin{cases}1, & \text { if } y<1 \\ e^{x}, & \text { if } y=1\end{cases}
$$

Then $X$ is a connected nonquasiconvex space and $\mu$ is doubling for balls of radii at most $R_{0}$ if and only if $R_{0} \leq \frac{1}{2}$. This shows that the quasiconvexity assumption in Proposition 3.2 cannot be dropped. 
Before proving Proposition 3.2 we deduce the following lemmas. In particular, Lemma 3.5 covers Proposition 3.2 under the extra assumption that $Y$ is a length space, but with better control of the doubling constant than what is possible in general quasiconvex spaces.

Lemma 3.4 Assume that $v$ is doubling on $Y$ for balls of radii at most $R_{0}$, with a doubling constant $C_{d}$. Then every ball $B$ of radius $r \leq \frac{7}{4} R_{0}$ can be covered by at most $C_{d}^{7}$ balls with centers in $B$ and radius $\frac{1}{7} r$.

Proof Find a maximal pairwise disjoint collection of balls $B_{j}$ with centers in $B$ and radii $\frac{1}{14} r$. Note that for each $j$,

$$
B_{j} \subset \frac{15}{14} B \text { and } \frac{15}{112} B \subset \frac{127}{112} \cdot 14 B_{j} \subset 16 B_{j}
$$

The doubling property then implies that

$$
v\left(\frac{15}{14} B\right) \leq C_{d}^{3} v\left(\frac{15}{112} B\right) \leq C_{d}^{7} v\left(B_{j}\right) .
$$

From this and the pairwise disjointness of all $B_{j}$ we thus obtain

$$
v\left(\frac{15}{14} B\right) \geq \sum_{j} v\left(B_{j}\right) \geq \frac{1}{C_{d}^{7}} v\left(\frac{15}{14} B\right) \sum_{j} 1,
$$

i.e. there are at most $C_{d}^{7}$ such balls. As the balls $2 B_{j}$ cover $B$, we are done.

Lemma 3.5 Assume that $Y$ is a length space and that $v$ is doubling on $Y$ for balls of radii at most $R_{0}$, with a doubling constant $C_{d}$. Let $n$ be a positive integer. Then the following are true:

(a) If $x, x^{\prime} \in Y, 0<r \leq R_{0}$ and $d\left(x, x^{\prime}\right)<n r$, then

$$
v\left(B\left(x^{\prime}, r\right)\right) \leq C_{d}^{n} v(B(x, r)) .
$$

(b) Every ball $B$ of radius $n r$, with $r \leq \frac{1}{4} R_{0}$, can be covered by at most $C_{d}^{7(n+4) / 6}$ balls of radius $r, n=1,2, \ldots$

In particular, for any $R_{1}>0, v$ is doubling on $Y$ for balls of radii at most $R_{1}$ with a doubling constant depending only on $R_{1} / R_{0}$ and $C_{d}$.

Proof (a) Connect $x$ and $x^{\prime}$ by a curve of length $l_{\gamma}<n r$. Along this curve, we can find balls $B_{j}$ of radius $r, j=0,1, \ldots, n$, such that $B_{0}=B(x, r), B_{n}=B\left(x^{\prime}, r\right)$ and $B_{j} \subset 2 B_{j-1}$. An iteration of the doubling property gives the desired estimate.

(b) Assume that $\varphi(n)$ is the smallest number such that each ball $B(x, n r)$ is covered by $\varphi(n)$ balls $B_{j}$ of radius $r$. As $Y$ is a length space, the balls $7 B_{j} \operatorname{cover} B(x,(n+6) r)$. Using Lemma 3.4, each $7 B_{j}$ can in turn be covered by at most $C_{d}^{7}$ balls of radius $r$, which implies that $\varphi(n+6) \leq C_{d}^{7} \varphi(n)$. Since $\varphi(1)=1$ and $\varphi$ is nondecreasing, the statement follows by induction. 
Proof of Proposition 3.2 We will use the inner metric $d_{\text {in }}$, defined in (2.1), and denote balls with respect to $d_{\text {in }}$ by $B_{\text {in }}$. It follows from the inclusions

$$
B_{\text {in }}(x, r) \subset B(x, r) \subset B_{\text {in }}(x, L r),
$$

together with a repeated use of the doubling property for metric balls, that $v$ is doubling for inner balls of radii at most $R_{0}$. As $\left(X, d_{\text {in }}\right)$ is a length space, it thus follows from Lemma 3.5 that $v$ is doubling for inner balls of radii at most $L R_{1}$. Hence, using the inclusions (3.1) again, $v$ is doubling for metric balls of radii at most $R_{1}$.

\section{The Measure $\mu_{\beta}$ is Globally Doubling on $X_{\varepsilon}$}

Standing assumptions for this section will be given after Example 4.3.

Given a uniformly locally doubling measure $\mu$ on the Gromov hyperbolic space $X$, we wish to obtain a globally doubling measure on its uniformization $X_{\varepsilon}$. We do so as follows.

Definition 4.1 Assume that $X$ is a locally compact roughly starlike Gromov hyperbolic space equipped with a Borel measure $\mu$, and that $z_{0} \in X$.

Fix $\beta>0$, and set $\mu_{\beta}$ to be the measure on $X=X_{\varepsilon}$ given by

$$
\mathrm{d} \mu_{\beta}=\rho_{\beta} \mathrm{d} \mu, \quad \text { where } \rho_{\beta}(x)=e^{-\beta d\left(x, z_{0}\right)} .
$$

We also extend this measure to $\bar{X}_{\varepsilon}$ by letting $\mu_{\beta}\left(\bar{X}_{\varepsilon} \backslash X_{\varepsilon}\right)=0$.

Our aim in this section is to show that $\mu_{\beta}$ is a globally doubling measure on $\bar{X}_{\varepsilon}$, under suitable assumptions (see Theorem 4.9).

Bonk-Heinonen-Koskela [14, Theorem 1.1] showed that there is a kind of duality between Gromov hyperbolic spaces and bounded uniform domains, see the introduction for further details. Here we also equip these spaces with measures. The following examples illustrate what happens in a simple case.

Example 4.2 The Euclidean real line $X=\mathbf{R}$ is Gromov hyperbolic, because it is a metric tree. Since $\delta=0$, any $\varepsilon>0$ is allowed in the uniformization process, by Theorem 2.6. Setting $z_{0}=0$, we now determine what $X_{\varepsilon}$ is. For $x, y \in \mathbf{R}$, the uniformized metric is given by

$$
d_{\varepsilon}(x, y)=\left|\int_{x}^{y} e^{-\varepsilon|t|} \mathrm{d} t\right|= \begin{cases}\frac{1}{\varepsilon}\left|e^{-\varepsilon|x|}-e^{-\varepsilon|y|}\right|, & \text { if } x y \geq 0, \\ \frac{1}{\varepsilon}\left(2-\left(e^{-\varepsilon|x|}+e^{-\varepsilon|y|}\right)\right), & \text { if } x y \leq 0 .\end{cases}
$$

With $y=0$ we get $d_{\varepsilon}(x, 0)=\left(1-e^{-\varepsilon|x|}\right) / \varepsilon$. Hence the map $\Phi: X_{\varepsilon} \rightarrow(-1 / \varepsilon, 1 / \varepsilon)$ given by

$$
\Phi(x)=\frac{1}{\varepsilon}\left(1-e^{-\varepsilon|x|}\right) \operatorname{sign} x
$$


is an isometry, identifying $X_{\varepsilon}$ with the open interval $(-1 / \varepsilon, 1 / \varepsilon)$.

However, when $X$ is equipped with the Lebesgue measure $\mathcal{L}^{1}$, the measure $\mu_{\beta}$ is not the Lebesgue measure on $(-1 / \varepsilon, 1 / \varepsilon)$. To determine $\mu_{\beta}$, note that it is absolutely continuous with respect to the Lebesgue measure $\mathcal{L}^{1}$ on the interval $(-1 / \varepsilon, 1 / \varepsilon)$. So we compute the Radon-Nikodym derivative of $\mu_{\beta}$ with respect to $\mathcal{L}^{1}$. By symmetry, it suffices to consider $x>0$. Then

$$
\mathrm{d} \mu_{\beta}(\Phi(x))=e^{-\beta x} J_{\Phi^{-1}(\Phi(x))} \mathrm{d} \mathcal{L}^{1}(\Phi(x))=e^{(\varepsilon-\beta) x} \mathrm{~d} \mathcal{L}^{1}(\Phi(x)) .
$$

Substituting $\Phi(x)=z$ in the above, we get

$$
\mathrm{d} \mu_{\beta}(z)=(1-\varepsilon z)^{-1+\beta / \varepsilon} \mathrm{d} \mathcal{L}^{1}(z)=\left(\varepsilon d_{\varepsilon}(z)\right)^{-1+\beta / \varepsilon} \mathrm{d} \mathcal{L}^{1}(z),
$$

where $d_{\varepsilon}(z)=1 / \varepsilon-z$ is the distance from $\Phi(x)=z \geq 0$ to the boundary $\{ \pm 1 / \varepsilon\}$ of $\Phi\left(X_{\varepsilon}\right)$.

Similarly, if $X=\mathbf{R}$ is equipped with a weighted measure

$$
\mathrm{d} \mu(x)=w(x) \mathrm{d} \mathcal{L}^{1}(x)
$$

then as in (4.1),

$$
\mathrm{d} \mu_{\beta}(z)=\left(\varepsilon d_{\varepsilon}(z)\right)^{-1+\beta / \varepsilon} w\left(\Phi^{-1}(z)\right) \mathrm{d} \mathcal{L}^{1}(z) .
$$

The following example reverses the procedure in Example 4.2.

Example 4.3 The interval $X=(-1,1)$ is a uniform domain and so, by Theorem 3.6 in Bonk-Heinonen-Koskela [14], it becomes a Gromov hyperbolic space when equipped with the quasihyperbolic metric $k$. The quasihyperbolic metric is for $0 \leq y<z<1$ given by

$$
k(y, z)=\int_{y}^{z} \frac{1}{1-t} \mathrm{~d} t=\log \left(\frac{1-y}{1-z}\right),
$$

cf. Sect. 7. With $z_{0}=0$, by symmetry, we have $k\left(z, z_{0}\right)=\log (1 /(1-|z|))$ for $z \in X$. Hence we consider the map $\Psi:(-1,1) \rightarrow \mathbf{R}$ given by

$$
\Psi(z)=(\operatorname{sign} z) \log \frac{1}{1-|z|},
$$

and see that $\Psi$ is an isometry between the Gromov hyperbolic space $(X, k)$ and the Euclidean line $\mathbf{R}$. By Example 4.2 with $\varepsilon=1$, the uniformization of $\mathbf{R}$ gives back the Euclidean interval $(-1,1)$.

We wish to find a measure $\mu$ on $(X, k)=\mathbf{R}$ such that the weighted measure $\mu_{\beta}$ given by Definition 4.1 becomes the Lebesgue measure on $(-1,1)$. In view of (4.2) with $\varepsilon=1$ and $\Phi=\Psi^{-1}, \mu$ is given by $\mathrm{d} \mu(x)=w(x) \mathrm{d} \mathcal{L}^{1}(x)$, where

$$
w(x)=d_{1}(\Phi(x))^{1-\beta}=(1-|\Phi(x)|)^{1-\beta}=e^{(\beta-1)|x|} .
$$


In the rest of this section, we assume that $X$ is a locally compact roughly starlike Gromov $\delta$-hyperbolic space equipped with a measure $\mu$ which is doubling on $X$ for balls of radii at most $R_{0}$, with a doubling constant $C_{d}$. We also fix a point $z_{0} \in X$, let $M$ be the constant in the roughly starlike condition with respect to $z_{0}$, and assume that

$$
0<\varepsilon \leq \varepsilon_{0}(\delta) \text { and } \beta>\beta_{0}:=\frac{17 \log C_{d}}{3 R_{0}}
$$

Finally, we let $X_{\varepsilon}$ be the uniformization of $X$ with uniformization center $z_{0}$.

In specific cases one may want to consider how to optimally choose $R_{0}$, and the corresponding $C_{d}$, in the formula for $\beta_{0}$. The factor $\frac{17}{3}$ comes from various estimates leading up to the proof of Proposition 4.7, and is not likely to be optimal. The following example shows however that it is not too far from optimal and that it cannot be replaced by any constant $<1$.

Example 4.4 Let $X$ be the infinite regular $K$-ary metric tree, equipped with the Lebesgue measure $\mu$, as in Björn-Björn-Gill-Shanmugalingam [8, Sect. 3]. Since it is a tree, any $\varepsilon>0$ is allowed for uniformization.

If $C_{d}(R)$ is the optimal doubling constant for radii $\leq R$, then a straightforward calculation shows that

$$
\lim _{R \rightarrow \infty} \frac{C_{d}(R)}{K^{R}}=1
$$

and thus we are allowed, in this paper, to use any

$$
\beta>\frac{17 \log K^{R}}{3 R}=\frac{17}{3} \log K .
$$

In this specific case, it was shown in [8, Corollary 3.9] that $\mu_{\beta}$ is globally doubling and supports a global 1-Poincaré inequality on $X_{\varepsilon}$ whenever $\beta>\log K$. For $\beta \leq \log K$, $\mu_{\beta}\left(X_{\varepsilon}\right)=\infty$ and $\mu_{\beta}$ cannot possibly be globally doubling on the bounded space $X_{\varepsilon}$.

The following lemma gives us an estimate of $\mu_{\beta}(B)$ for subWhitney balls $B$.

Lemma 4.5 Let $x \in X$ and $0<r \leq \frac{1}{2} d_{\varepsilon}(x)$. Then

$$
\mu_{\beta}\left(B_{\varepsilon}(x, r)\right) \simeq \rho_{\beta}(x) \mu\left(B\left(x, \frac{r}{\rho_{\varepsilon}(x)}\right)\right)
$$

with comparison constants depending only on $M, \varepsilon, C_{d}, R_{0}$ and $\beta$.

Proof By Lemma 2.8, we have for all $y \in B_{\varepsilon}(x, r)$,

$$
\rho_{\beta}(y)=\rho_{\varepsilon}(y)^{\beta / \varepsilon} \simeq\left(\varepsilon d_{\varepsilon}(y)\right)^{\beta / \varepsilon} \simeq\left(\varepsilon d_{\varepsilon}(x)\right)^{\beta / \varepsilon} \simeq \rho_{\beta}(x) .
$$


Moreover, Theorem 2.10 implies that

$$
B\left(x, \frac{C_{1} r}{\rho_{\varepsilon}(x)}\right) \subset B_{\varepsilon}(x, r) \subset B\left(x, \frac{C_{2} r}{\rho_{\varepsilon}(x)}\right) .
$$

This yields

$$
\mu_{\beta}\left(B_{\varepsilon}(x, r)\right) \simeq \rho_{\beta}(x) \mu\left(B_{\varepsilon}(x, r)\right) \lesssim \rho_{\beta}(x) \mu\left(B\left(x, \frac{C_{2} r}{\rho_{\varepsilon}(x)}\right)\right)
$$

and similarly, $\mu_{\beta}\left(B_{\varepsilon}(x, r)\right) \gtrsim \rho_{\beta}(x) \mu\left(B\left(x, C_{1} r / \rho_{\varepsilon}(x)\right)\right.$. Finally, Lemma 3.5 shows that the last two balls in $X$ have measure comparable to $\mu\left(B\left(x, r / \rho_{\varepsilon}(x)\right)\right.$, which concludes the proof.

Remark 4.6 Lemma 4.5 implies that if $\mu_{\beta}$ is globally doubling on $X_{\varepsilon}$ then $\mu$ is uniformly locally doubling on $X$, i.e. the converse of Theorem 1.1 (a) holds. Indeed, if $0<r \leq \frac{1}{4 e \varepsilon}$ and $x \in X$, then $2 r \rho_{\varepsilon}(x) \leq \frac{1}{2} d_{\varepsilon}(x)$, by (2.3). Lemma 4.5, with $r$ replaced by $2 r \rho_{\varepsilon}(x)$ and $r \rho_{\varepsilon}(x)$, respectively, then gives

$$
\mu(B(x, 2 r)) \simeq \frac{\mu_{\beta}\left(B_{\varepsilon}\left(x, 2 r \rho_{\varepsilon}(x)\right)\right)}{\rho_{\beta}(x)} \simeq \frac{\mu_{\beta}\left(B_{\varepsilon}\left(x, r \rho_{\varepsilon}(x)\right)\right)}{\rho_{\beta}(x)} \simeq \mu(B(x, r)) .
$$

Similar arguments, combined with the arguments in the proof of Lemma 6.1, show that if $\mu_{\beta}$ also supports a global $p$-Poincaré inequality on $X_{\varepsilon}$ or $\bar{X}_{\varepsilon}$ then $\mu$ supports a uniformly local $p$-Poincaré inequality on $X$, i.e. the converse of Theorem 1.1 (b) holds.

We shall now estimate $\mu_{\beta}(B)$ for balls $B$ centered at $\partial_{\varepsilon} X$ in terms of the (essentially) largest Whitney ball contained in $B$. The existence of such balls is given by Lemma 4.8 below.

Proposition 4.7 Let $\xi \in \partial_{\varepsilon} X$ and $0<r \leq 2 \operatorname{diam}_{\varepsilon} X_{\varepsilon}$. Assume that $a_{0}>0$ and $z \in X$ are such that $B_{\varepsilon}\left(z, a_{0} r\right) \subset B_{\varepsilon}(\xi, r)$ and $d_{\varepsilon}(z) \geq 2 a_{0} r$. Then,

$$
\mu_{\beta}\left(B_{\varepsilon}(\xi, r)\right) \simeq \rho_{\beta}(z) \mu\left(B\left(z, R_{0}\right)\right) \simeq \rho_{\beta}(z) \mu\left(B\left(z, \frac{a_{0} r}{\rho_{\varepsilon}(z)}\right)\right) \text { and } \rho_{\beta}(z) \simeq(\varepsilon r)^{\beta / \varepsilon},
$$

where the comparison constants depend only on $\delta, M, \varepsilon, C_{d}, R_{0}, \beta$ and $a_{0}$.

Proof For $n=1,2, \ldots$, define the boundary layers

$$
A_{n}=\left\{x \in B_{\varepsilon}(\xi, r): e^{-n} r \leq d_{\varepsilon}(x) \leq e^{1-n} r\right\}
$$

Corollary 2.9 implies that for every $x \in A_{n}$, either $\varepsilon d(x, z)<1$ or

$$
\exp (\varepsilon d(x, z)) \simeq \frac{d_{\varepsilon}(x, z)^{2}}{d_{\varepsilon}(x) d_{\varepsilon}(z)} \leq \frac{\left(d_{\varepsilon}(x, \xi)+d_{\varepsilon}(\xi, z)\right)^{2}}{2 a_{0} e^{-n} r^{2}} \leq \frac{2 e^{n}}{a_{0}}
$$


and hence $\varepsilon d(x, z)<n+C$, where $C$ depends only on $\delta, M, \varepsilon$ and $a_{0}$.

Using Lemma $3.5(\mathrm{~b})$, we can thus cover each layer $A_{n} \subset B(z,(n+C) / \varepsilon)$ by $N_{n} \lesssim C_{d}^{14 n / 3 \varepsilon R_{0}}$ balls $B_{n, j}$ with centers in $B(z,(n+C) / \varepsilon)$ and radius $R_{0}$. Since $X_{\varepsilon}$ is geodesic, Lemma 3.5 (a) implies that each of these balls satisfies

$$
\mu\left(B_{n, j}\right) \lesssim C_{d}^{n / \varepsilon R_{0}} \mu\left(B\left(z, R_{0}\right)\right)
$$

Moreover, as in (4.4) we see that $\rho_{\beta}(z)=\rho_{\varepsilon}(z)^{\beta / \varepsilon} \simeq\left(\varepsilon d_{\varepsilon}(z)\right)^{\beta / \varepsilon} \simeq(\varepsilon r)^{\beta / \varepsilon}$ and

$$
\rho_{\beta}(x)=\rho_{\varepsilon}(x)^{\beta / \varepsilon} \simeq\left(\varepsilon d_{\varepsilon}(x)\right)^{\beta / \varepsilon} \simeq\left(e^{-n} \varepsilon r\right)^{\beta / \varepsilon} \text { for all } x \in A_{n} .
$$

It thus follows that

$$
\mu_{\beta}\left(A_{n} \cap B_{n, j}\right) \lesssim\left(e^{-n} \varepsilon r\right)^{\beta / \varepsilon} \mu\left(B_{n, j}\right) \lesssim C_{d}^{n / \varepsilon R_{0}} e^{-n \beta / \varepsilon} \rho_{\beta}(z) \mu\left(B\left(z, R_{0}\right)\right)
$$

and hence for $\beta>\beta_{0}=17\left(\log C_{d}\right) / 3 R_{0}$,

$$
\begin{aligned}
\mu_{\beta}\left(B_{\varepsilon}(\xi, r)\right) \leq & \sum_{n=1}^{\infty} \sum_{j=1}^{N_{n}} \mu_{\beta}\left(A_{n} \cap B_{n, j}\right) \\
& \lesssim \rho_{\beta}(z) \mu\left(B\left(z, R_{0}\right)\right) \sum_{n=1}^{\infty}\left(C_{d}^{17 / 3 R_{0}}\right)^{n / \varepsilon} e^{-n \beta / \varepsilon} \simeq \rho_{\beta}(z) \mu\left(B\left(z, R_{0}\right)\right)
\end{aligned}
$$

Since $a_{0} r \leq \frac{1}{2} d_{\varepsilon}(z)$, Lemma 4.5 implies that

$$
\mu_{\beta}\left(B_{\varepsilon}(\xi, r)\right) \geq \mu_{\beta}\left(B_{\varepsilon}\left(z, a_{0} r\right)\right) \simeq \rho_{\beta}(z) \mu\left(B\left(z, \frac{a_{0} r}{\rho_{\varepsilon}(z)}\right)\right) .
$$

By (2.3) we see that

$$
r>d_{\varepsilon}(z) \geq \frac{\rho_{\varepsilon}(z)}{e \varepsilon}
$$

and hence, by the doubling property for $\mu$ on $X$,

$$
\mu\left(B\left(z, \frac{a_{0} r}{\rho_{\varepsilon}(z)}\right)\right) \geq \mu\left(B\left(z, \frac{a_{0}}{e \varepsilon}\right)\right) \simeq \mu\left(B\left(z, R_{0}\right)\right) .
$$

The following lemma shows how to pick $z$ and $a_{0}$ in Proposition 4.7.

Lemma 4.8 Let $0<a_{0}<a:=\min \left\{\frac{1}{8}, \frac{1}{6 A}\right\}$, where $A=A(\delta)$ is as in Theorem 2.6. Then for every $x \in \bar{X}_{\varepsilon}$ and every $0<r \leq 2 \operatorname{diam}_{\varepsilon} X_{\varepsilon}$ we can find a ball $B_{\varepsilon}\left(z, a_{0} r\right) \subset$ $B_{\varepsilon}(x, r)$ such that $d_{\varepsilon}(z) \geq 2 a_{0} r$. 
Proof First, assume that $x \in X_{\varepsilon}$. By Theorem 2.6, there is an $A$-uniform curve $\gamma$ from $x$ to $z_{0}$, parametrized by arc length $\mathrm{d} s_{\varepsilon}$. If $l_{\varepsilon}(\gamma) \geq \frac{2}{3} r$ then for $z=\gamma\left(\frac{1}{3} r\right)$ we have

$$
d_{\varepsilon}(z) \geq \frac{r}{3 A} \text { and } \quad B_{\varepsilon}\left(z, \frac{r}{6 A}\right) \subset B_{\varepsilon}\left(x, \frac{r}{3}+\frac{r}{6 A}\right) \subset B_{\varepsilon}(x, r) .
$$

Thus, any $a_{0} \leq \frac{1}{6 A}$ will do in this case. If $l_{\varepsilon}(\gamma)<\frac{2}{3} r$, then letting $z=z_{0}$ yields

$$
B_{\varepsilon}\left(z, \frac{1}{3} r\right) \subset B_{\varepsilon}\left(x, l_{\varepsilon}(\gamma)+\frac{1}{3} r\right) \subset B_{\varepsilon}(x, r),
$$

and for $a_{0} \leq \frac{1}{8}$

$$
d_{\varepsilon}(z)=d_{\varepsilon}\left(z_{0}\right) \geq 4 a_{0} \operatorname{diam}_{\varepsilon} X_{\varepsilon} \geq 2 a_{0} r .
$$

This proves the lemma for $x \in X_{\varepsilon}$. For $x \in \partial_{\varepsilon} X$ and any $0<a_{0}<a$, choose $r^{\prime}=a_{0} r / a$ and $x^{\prime} \in X_{\varepsilon}$ sufficiently close to $x$ so that, with the corresponding $z$,

$$
B_{\varepsilon}\left(z, a_{0} r\right)=B_{\varepsilon}\left(z, a r^{\prime}\right) \subset B_{\varepsilon}\left(x^{\prime}, r^{\prime}\right) \subset B(x, r) \text { and } d_{\varepsilon}(z) \geq 2 a r^{\prime}=2 a_{0} r .
$$

Lemma 4.5 and Proposition 4.7 can be summarized in the following result, which roughly says that in $\left(X_{\varepsilon}, \mu_{\beta}\right)$, the measure of every ball is comparable to the measure of the (essentially) largest Whitney ball contained in it.

Theorem 4.9 The measure $\mu_{\beta}$ is globally doubling on $\bar{X}_{\varepsilon}$.

Moreover, with $a_{0}$ and $z$ provided by Lemma 4.8, we have for every $x \in \bar{X}_{\varepsilon}$ and $0<r \leq 2 \operatorname{diam}_{\varepsilon} X_{\varepsilon}$,

$$
\mu_{\beta}\left(B_{\varepsilon}(x, r)\right) \simeq \mu_{\beta}\left(B_{\varepsilon}\left(z, a_{0} r\right)\right),
$$

where the comparison constants depend only on $\delta, M, \varepsilon, C_{d}, R_{0}, \beta$ and $a_{0}$.

It follows directly that $\mu_{\beta}$ is globally doubling also on $X_{\varepsilon}$. The optimal doubling constants are the same, by Proposition 3.3 in Björn-Björn [7].

Proof We start by proving the measure estimate (4.6). As $a_{0} r \leq \frac{1}{2} d_{\varepsilon}(z)$, Lemma 4.5 applied to $B_{\varepsilon}\left(z, a_{0} r\right)$ implies that

$$
\mu_{\beta}\left(B_{\varepsilon}\left(z, a_{0} r\right)\right) \simeq \rho_{\beta}(z) \mu\left(B\left(z, \frac{a_{0} r}{\rho_{\varepsilon}(z)}\right)\right) .
$$

If $0<r \leq \frac{1}{2} d_{\varepsilon}(x)$ then by (4.4), (4.5) and Lemma 2.8,

$$
\rho_{\beta}(z) \simeq \rho_{\beta}(x) \text { and } d(x, z) \leq \frac{C_{2} r}{\rho_{\varepsilon}(x)} \lesssim \frac{1}{2}
$$


Lemma 3.5 then implies that

$$
\mu\left(B\left(z, \frac{a_{0} r}{\rho_{\varepsilon}(z)}\right)\right) \simeq \mu\left(B\left(z, \frac{r}{\rho_{\varepsilon}(z)}\right)\right) \simeq \mu\left(B\left(x, \frac{r}{\rho_{\varepsilon}(x)}\right)\right),
$$

and another application of Lemma 4.5, this time to $B_{\varepsilon}(x, r)$, proves (4.6) in this case.

If $r \geq \frac{1}{2} d_{\varepsilon}(x)$ then $B_{\varepsilon}(x, r) \subset B_{\varepsilon}(\xi, 3 r)$ for some $\xi \in \partial_{\varepsilon} X$. Proposition 4.7 (with $a_{0}$ replaced by $\left.\frac{1}{3} a_{0}\right)$ then implies

$$
\mu_{\beta}\left(B_{\varepsilon}(\xi, 3 r)\right) \simeq \rho_{\beta}(z) \mu\left(B\left(z, \frac{a_{0} r}{\rho_{\varepsilon}(z)}\right)\right),
$$

which together with (4.7) proves (4.6) also in this case.

To conclude the doubling property, use the Whitney ball $B_{\varepsilon}\left(z, a_{0} r\right)$ for both $B_{\varepsilon}(x, r)$ and $B_{\varepsilon}(x, 2 r)$, with constants $a_{0}$ and $a_{0}^{\prime}=\frac{1}{2} a_{0}$, respectively. Since

$$
d_{\varepsilon}(z) \geq 2 a_{0} r=2 a_{0}^{\prime} \cdot 2 r,
$$

we have by (4.6), first used with $a_{0}^{\prime}$ and then with $a_{0}$,

$$
\mu_{\beta}\left(B_{\varepsilon}(x, 2 r)\right) \simeq \mu_{\beta}\left(B_{\varepsilon}\left(z, 2 a_{0}^{\prime} r\right)\right)=\mu_{\beta}\left(B_{\varepsilon}\left(z, a_{0} r\right)\right) \simeq \mu_{\beta}\left(B_{\varepsilon}(x, r)\right) .
$$

We conclude this section with an estimate of the lower and upper dimensions for the measure $\mu_{\beta}$ at $\partial_{\varepsilon} X$.

Lemma 4.10 Let

$$
s_{ \pm}=\frac{\beta}{\varepsilon} \pm \frac{\log C_{d}}{\varepsilon R_{0}} .
$$

Then for all $\xi \in \partial_{\varepsilon} X$ and all $0<r \leq r^{\prime} \leq 2 \operatorname{diam}_{\varepsilon} X_{\varepsilon}$,

$$
\left(\frac{r}{r^{\prime}}\right)^{s_{+}} \lesssim \frac{\mu_{\beta}\left(B_{\varepsilon}(\xi, r)\right)}{\mu_{\beta}\left(B_{\varepsilon}\left(\xi, r^{\prime}\right)\right)} \lesssim\left(\frac{r}{r^{\prime}}\right)^{s_{-}}
$$

with comparison constants depending only on $\delta, M, \varepsilon, C_{d}, R_{0}, \beta$ and the constant $a_{0}$ from Theorem 2.6.

Note that $0<s_{-} \leq s_{+}$, because $\beta>\beta_{0}$, where $\beta_{0}$ is as in (4.3).

Proof Proposition 4.7 and Lemma 4.8 imply that there are $z, z^{\prime} \in X$ such that

$$
\begin{aligned}
\mu_{\beta}\left(B_{\varepsilon}(\xi, r)\right) & \simeq(\varepsilon r)^{\beta / \varepsilon} \mu\left(B\left(z, R_{0}\right)\right), \\
\mu_{\beta}\left(B_{\varepsilon}\left(\xi, r^{\prime}\right)\right) & \simeq\left(\varepsilon r^{\prime}\right)^{\beta / \varepsilon} \mu\left(B\left(z^{\prime}, R_{0}\right)\right),
\end{aligned}
$$

where

$$
\begin{array}{rlrl}
B_{\varepsilon}\left(z, a_{0} r\right) & \subset B_{\varepsilon}(\xi, r), & d_{\varepsilon}(z) \geq 2 a_{0} r \\
B_{\varepsilon}\left(z^{\prime}, a_{0} r^{\prime}\right) & \subset B_{\varepsilon}\left(\xi, r^{\prime}\right), & & d_{\varepsilon}\left(z^{\prime}\right) \geq 2 a_{0} r^{\prime} .
\end{array}
$$


From Corollary 2.9 we conclude that if $\varepsilon d\left(z, z^{\prime}\right) \geq 1$ then

$$
\exp \left(\varepsilon d\left(z, z^{\prime}\right)\right) \simeq \frac{d_{\varepsilon}\left(z, z^{\prime}\right)^{2}}{d_{\varepsilon}(z) d_{\varepsilon}\left(z^{\prime}\right)} \leq \frac{\left(2 r^{\prime}\right)^{2}}{\left(2 a_{0} r\right)\left(2 a_{0} r^{\prime}\right)}=\frac{r^{\prime}}{a_{0}^{2} r},
$$

and hence $d\left(z, z^{\prime}\right) \leq \frac{1}{\varepsilon}\left(C+\log \left(r^{\prime} / r\right)\right)$ holds regardless of the value of $d\left(z, z^{\prime}\right)$. Lemma 3.5 (a) with $n=\left\lceil d\left(z, z^{\prime}\right) / R_{0}\right\rceil$ (the smallest integer $\geq d\left(z, z^{\prime}\right) / R_{0}$ ) then implies that

$$
\frac{\mu\left(B\left(z, R_{0}\right)\right)}{\mu\left(B\left(z^{\prime}, R_{0}\right)\right)} \geq C_{d}^{-n} \gtrsim\left(\frac{r}{r^{\prime}}\right)^{\left(\log C_{d}\right) / \varepsilon R_{0}},
$$

which together with (4.8) proves the first inequality in the lemma. The second inequality follows similarly by interchanging $z$ and $z^{\prime}$ in (4.9).

\section{Upper Gradients and Poincaré Inequalities}

We assume in this section that $1 \leq p<\infty$ and that $Y=(Y, d, v)$ is a metric space equipped with a complete Borel measure v such that $0<v(B)<\infty$ for all balls $B \subset Y$.

We follow Heinonen and Koskela [29] in introducing upper gradients as follows (in [29] they are referred to as very weak gradients).

Definition 5.1 A Borel function $g: Y \rightarrow[0, \infty]$ is an upper gradient of an extended real-valued function $u$ on $Y$ if for all arc length parametrized curves $\gamma:\left[0, l_{\gamma}\right] \rightarrow Y$,

$$
\left|u(\gamma(0))-u\left(\gamma\left(l_{\gamma}\right)\right)\right| \leq \int_{\gamma} g \mathrm{~d} s
$$

where we follow the convention that the left-hand side is considered to be $\infty$ whenever at least one of the terms therein is $\pm \infty$. If $g$ is a nonnegative measurable function on $Y$ and if (5.1) holds for $p$-almost every curve (see below), then $g$ is a $p$-weak upper gradient of $u$.

We say that a property holds for $p$-almost every curve if it fails only for a curve family $\Gamma$ with zero $p$-modulus, i.e. there is a Borel function $0 \leq \rho \in L^{p}(Y)$ such that $\int_{\gamma} \rho \mathrm{d} s=\infty$ for every curve $\gamma \in \Gamma$. The $p$-weak upper gradients were introduced in Koskela-MacManus [34]. It was also shown therein that if $g \in L_{\text {loc }}^{p}(Y)$ is a $p$-weak upper gradient of $u$, then one can find a sequence $\left\{g_{j}\right\}_{j=1}^{\infty}$ of upper gradients of $u$ such that $\left\|g_{j}-g\right\|_{L^{p}(Y)} \rightarrow 0$.

If $u$ has an upper gradient in $L_{\text {loc }}^{p}(Y)$, then it has a minimal $p$-weak upper gradient $g_{u} \in L_{\mathrm{loc}}^{p}(Y)$ in the sense that for every $p$-weak upper gradient $g \in L_{\mathrm{loc}}^{p}(Y)$ of $u$ we have $g_{u} \leq g$ a.e., see Shanmugalingam [37] (or [5] or [30]). The minimal $p$-weak upper gradient is well-defined up to a set of measure zero. 
Definition 5.2 $Y$ (or $v$ ) supports a global $p$-Poincaré inequality if there exist constants $\lambda \geq 1$ (called dilation) and $C_{\mathrm{PI}}>0$ such that for all balls $B \subset Y$, all bounded measurable functions $u$ on $Y$ and all upper gradients $g$ of $u$,

$$
f_{B}\left|u-u_{B}\right| \mathrm{d} v \leq C_{\mathrm{PI}} \operatorname{diam}(B)\left(f_{\lambda B} g^{p} \mathrm{~d} v\right)^{1 / p},
$$

where $u_{B}:=u_{B, v}:=f_{B} u \mathrm{~d} v:=\int_{B} u \mathrm{~d} v / v(B)$.

If this holds only for balls $B$ of radii $\leq R_{0}$, then we say that $v$ supports a $p$-Poincaré inequality for balls of radii at most $R_{0}$, and also that $Y$ (or $v$ ) supports a uniformly local p-Poincaré inequality.

Multiplying bounded measurable functions by suitable cut-off functions and truncating integrable functions shows that one may replace "bounded measurable" by "integrable" in the definition. On the other hand, the proofs of [30, Lemma 8.1.5 and Theorem 8.1.53] show that (5.2) can equivalently be required for all (not necessarily bounded) measurable functions $u$ on $\lambda B$ and all upper (or $p$-weak upper) gradients $g$ of $u$. See also [5, Proposition 4.13], [30, Theorem 8.1.49], Hajłasz-Koskela [26, Theorem 3.2], Heinonen-Koskela [29, Lemma 5.15] and Keith [32, Theorem 2] for further equivalent versions.

Theorem 5.3 Assume that $v$ is doubling and supports a p-Poincaré inequality, both properties holding for balls of radii at most $R_{0}$. Also assume that $Y$ is $L$-quasiconvex and that $R_{1}>0$.

Then $v$ supports a p-Poincaré inequality, with dilation constant $L$, for balls of radii at most $R_{1}$.

The proof below can be easily adapted to show that the same is true for so-called $(q, p)$-Poincare inequalities. The following examples show that the quasiconvexity assumption cannot be dropped even if one assumes that $v$ is globally doubling, and that one cannot replace $L$ in the conclusion by the dilation constant in the assumed $p$-Poincaré inequality, nor any fixed multiple of it.

Example 5.4 Let $X=([0, \infty) \times\{0,1\}) \cup(\{0\} \times[0,1])$ equipped with the Euclidean distance and the Lebesgue measure $\mathcal{L}^{1}$. Then $X$ is a connected nonquasiconvex space and $\mathcal{L}^{1}$ is globally doubling on $X$. However, $\mathcal{L}^{1}$ supports a $p$-Poincaré inequality on $X, p \geq 1$, for balls of radii at most $R_{0}$ if and only if $R_{0} \leq 1$. In this case one can choose the dilation constant $\lambda=1$. This shows that the quasiconvexity assumption in Theorem 5.3 cannot be dropped.

Example 5.5 For $a \geq 1$, let $X=([0, a] \times\{0,1\}) \cup(\{0\} \times[0,1])$, equipped with the Euclidean distance and the Lebesgue measure $\mathcal{L}^{1}$. Then $X$ is a connected $(2 a+1)$ quasiconvex space and $\mathcal{L}^{1}$ is globally doubling on $X$. In this case, $\mathcal{L}^{1}$ supports a $p$-Poincaré inequality on $X, p \geq 1$, for balls of radii at most $R_{0}$ for any $R_{0}>0$, with the optimal dilation

$$
\begin{cases}1, & \text { if } R_{0} \leq 1 \\ \sqrt{1+a^{2}}, & \text { if } R_{0}>1\end{cases}
$$


This shows that the dilation constant $L$ in the conclusion of Theorem 5.3 cannot in general be replaced by the dilation constant in the $p$-Poincaré inequality assumed for balls $\leq R_{0}$, nor any fixed multiple of it.

Proof of Theorem 5.3 The arguments are similar to the proof of Theorem 4.4 in BjörnBjörn [6]. Let $C_{d}, C_{\mathrm{PI}}$ and $\lambda$ be the constants in the doubling property and the $p$ Poincaré inequality for balls of radii $\leq R_{0}$. Let $B$ be a ball of radius $r_{B} \leq \frac{5}{2} L R_{1}=: R_{2}$. We can assume that $r_{B}>R_{0}$.

First, note that the conclusions in the first paragraph of the proof in [6] with $B_{0}=B$, $\sigma=L, r^{\prime}=R_{0} / \lambda$ and $\mu$ replaced by $\nu$, follow directly from our assumptions, without appealing to Lemma 4.7 nor Proposition 4.8 in [6]. This and the use of Lemma 3.4 explains why there is no need to assume properness here.

By Lemma 3.5, $v$ is doubling for balls of radii $\leq 7 L R_{2}$, with doubling constant $C_{d}^{\prime}$, depending only on $C_{d}$ and $L R_{2} / R_{0}$. Hence, using Lemma 3.4, we can cover $B$ by at most $\left(C_{d}^{\prime}\right)^{7\left\lceil\log _{7}\left(R_{2} / r^{\prime}\right)\right\rceil}$ balls $B_{j}^{\prime}$ with radius $r^{\prime}$. Their centers can then be connected by $L$-quasiconvex curves. As in the proof of [6, Theorem 4.4], we then construct along these curves a chain $\left\{B_{j}\right\}_{j=1}^{N}$ of balls of radius $r^{\prime}$, covering $B$ and with a uniform bound on $N$. It follows that the constant $C^{\prime \prime}$ in the proof of [6, Theorem 4.4] only depends on $C_{d}, C_{\mathrm{PI}}, \lambda, L$ and $R_{2} / R_{0}$. Thus we conclude from the last but one displayed formula in the proof of [6, Theorem 4.4] (with $B_{0}=B$ ) that $v$ supports a $p$-Poincaré inequality for balls of radii at most $R_{2}$, with dilation $2 L$.

That we can replace $2 L$ by $L$ now follows from [6, Theorem 5.1], provided that we decrease the bound on the radii to $R_{1}$.

\section{Poincaré Inequality on $X_{\varepsilon}$}

In this section, we assume that $X$ is a locally compact roughly starlike Gromov $\delta$ hyperbolic space equipped with a Borel measure $\mu$. We also fix a point $z_{0} \in X$, let $M$ be the constant in the roughly starlike condition with respect to $z_{0}$, and assume that

$$
0<\varepsilon \leq \varepsilon_{0}(\delta) \text { and } 1 \leq p<\infty
$$

Finally, we let $X_{\varepsilon}$ be the uniformization of $X$ with uniformization center $z_{0}$.

The following lemma shows that the $p$-Poincaré inequality holds for $\mu_{\beta}$ on sufficiently small subWhitney balls in $X_{\varepsilon}$. Recall that $\beta_{0}=\left(17 \log C_{d}\right) / 3 R_{0}$ as in (4.3).

Lemma 6.1 Assume that $\mu$ is doubling, with constant $C_{d}$, and supports a p-Poincaré inequality, with constants $C_{\mathrm{PI}}$ and $\lambda$, both properties holding for balls of radii at most $R_{0}$. Let $\beta>\beta_{0}$.

Then there exists $c_{0}>0$, depending only on $\delta, M, \varepsilon, R_{0}$ and $\lambda$, such that for all $x \in X_{\varepsilon}$ and all $0<r \leq c_{0} d_{\varepsilon}(x)$, the $p$-Poincaré inequality for $\mu_{\beta}$ holds on $B_{\varepsilon}=B_{\varepsilon}(x, r)$, i.e. for all bounded measurable functions $u$ and upper gradients $g_{\varepsilon}$ of u on $X_{\varepsilon}$ we have

$$
f_{B_{\varepsilon}}\left|u-u_{B_{\varepsilon}, \mu_{\beta}}\right| \mathrm{d} \mu_{\beta} \leq C r\left(f_{\tau B_{\varepsilon}} g_{\varepsilon}^{p} \mathrm{~d} \mu_{\beta}\right)^{1 / p}
$$


where $\tau=C_{2} \lambda / C_{1}$, with $C_{1}$ and $C_{2}$ from Theorem 2.10, and $C$ depends only on $\delta$, $M, \varepsilon, C_{d}, R_{0}, \beta, \lambda$ and $C_{\mathrm{PI}}$.

Proof Theorem 2.10 shows that if $c_{0} \leq C_{1} / 2 C_{2} \lambda$ then

$$
B_{\varepsilon} \subset B\left(x, \frac{C_{2} r}{\rho_{\varepsilon}(x)}\right)=: B \subset \lambda B \subset B_{\varepsilon}\left(x, \frac{C_{2} \lambda r}{C_{1}}\right)=\tau B_{\varepsilon} .
$$

Moreover, as in (4.4) we have for all $y \in \tau B_{\varepsilon}$,

$$
\rho_{\beta}(y)=\rho_{\varepsilon}(y)^{\beta / \varepsilon} \simeq \rho_{\varepsilon}(x)^{\beta / \varepsilon}=\rho_{\beta}(x) .
$$

Hence, by Theorem 4.9, all the balls in (6.1) have comparable $\mu_{\beta}$-measures, as well as comparable $\mu$-measures.

Let $u$ be a bounded measurable function on $X_{\varepsilon}$, or equivalently on $X$, and let $g_{\varepsilon}$ be an upper gradient of $u$ on $X_{\varepsilon}$. Since the arc length parametrization $\mathrm{d} s_{\varepsilon}$ with respect to $d_{\varepsilon}$ satisfies $\mathrm{d} s_{\varepsilon}=\rho_{\varepsilon} \mathrm{d} s$, we conclude that for all compact rectifiable curves $\gamma$ in $X_{\varepsilon}$,

$$
\int_{\gamma} g_{\varepsilon} \mathrm{d} s_{\varepsilon}=\int_{\gamma} g_{\varepsilon} \rho_{\varepsilon} \mathrm{d} s
$$

and thus $g:=g_{\varepsilon} \rho_{\varepsilon}$ is an upper gradient of $u$ on $X$. (Note that a compact curve in $X$ is rectifiable with respect to $d$ if and only if it is rectifiable with respect to $d_{\varepsilon}$.) If $c_{0} \leq R_{0} \varepsilon / C_{2}\left(2 e^{\varepsilon_{0} M}-1\right)$, then by Lemma 2.8 ,

$$
\frac{C_{2} r}{\rho_{\varepsilon}(x)} \leq \frac{C_{2} c_{0} d_{\varepsilon}(x)}{\rho_{\varepsilon}(x)} \leq \frac{C_{2} c_{0}\left(2 e^{\varepsilon_{0} M}-1\right)}{\varepsilon} \leq R_{0},
$$

and thus the $p$-Poincaré inequality holds on $B$. Using (6.2) we then obtain

$$
\begin{aligned}
f_{B_{\varepsilon}}\left|u-u_{B, \mu}\right| \mathrm{d} \mu_{\beta} & \lesssim f_{B}\left|u-u_{B, \mu}\right| \mathrm{d} \mu \leq \frac{C_{\mathrm{PI}} C_{2} r}{\rho_{\varepsilon}(x)}\left(f_{\lambda B} g^{p} \mathrm{~d} \mu\right)^{1 / p} \\
& \simeq \frac{r}{\rho_{\varepsilon}(x)}\left(f_{\lambda B}\left(g_{\varepsilon} \rho_{\varepsilon}\right)^{p} \mathrm{~d} \mu_{\beta}\right)^{1 / p} \lesssim r\left(f_{\tau B_{\varepsilon}} g_{\varepsilon}^{p} \mathrm{~d} \mu_{\beta}\right)^{1 / p} .
\end{aligned}
$$

Finally, a standard argument based on the triangle inequality makes it possible to replace $u_{B, \mu}$ on the left-hand side by $u_{B_{\varepsilon}, \mu_{\beta}}$.

Bonk-Heinonen-Koskela [14, Sect. 6] proved that if $\Omega$ is a locally compact uniform space equipped with a measure $\mu$ such that $(\Omega, \mu)$ is uniformly $Q$-Loewner in subWhitney balls, then $\Omega$ is globally $Q$-Loewner, where $Q>1$. If $\mu$ is locally doubling with $\mu(B(x, r)) \gtrsim r^{Q}$ whenever $B(x, r)$ is a subWhitney ball, then the local $Q$-Loewner condition is equivalent to an analogous local $Q$-Poincaré inequality, see [29, Theorems 5.7 and 5.9].

We have shown above that the measure $\mu_{\beta}$ on the uniformized space $X_{\varepsilon}$ is globally doubling and supports a $p$-Poincaré inequality for subWhitney balls. Following the 
philosophy of [14, Theorem 6.4], the next theorem demonstrates that the $p$-Poincare inequality is actually global on $X_{\varepsilon}$.

Theorem 6.2 Assume that $\mu$ is doubling and supports a $p$-Poincaré inequality on $X$, both properties holding for balls of radii at most $R_{0}$. Let $\beta>\beta_{0}$ and $\lambda>1$.

Then $\mu_{\beta}$ is globally doubling and supports a global p-Poincaré inequality on $\bar{X}_{\varepsilon}$ with dilation 1 , and on $X_{\varepsilon}$ with dilation $\lambda$.

If $X_{\varepsilon}$ happens to be geodesic, then it follows from the proof below that we can choose the dilation constant $\lambda=1$ also on $X_{\varepsilon}$.

Proof The global doubling property follows from Theorem 4.9, both on $X_{\varepsilon}$ and $\bar{X}_{\varepsilon}$. Since $X_{\varepsilon}$ is a length space and Lemma 6.1 shows that the $p$-Poincaré inequality on $X_{\varepsilon}$ holds for subWhitney balls, the global $p$-Poincaré inequality on $X_{\varepsilon}$, with dilation $\lambda>1$, follows from the following proposition. Moreover, as $\bar{X}_{\varepsilon}$ is geodesic, the global $p$-Poincaré inequality on $\bar{X}_{\varepsilon}$, with dilation 1 , also follows from the following proposition.

Proposition 6.3 Let $(\Omega, d)$ be a bounded A-uniform space equipped with a globally doubling measure $v$, which supports a p-Poincaré inequality for all subWhitney balls corresponding to some fixed $0<c_{0}<1$. Assume that $\Omega$ is L-quasiconvex. Then $v$ supports a global p-Poincaré inequality on $\Omega$ with dilation $L$.

If moreover the completion $\bar{\Omega}$ is $L^{\prime}$-quasiconvex, then $v$, extended by $\nu(\partial \Omega)=0$, supports a global p-Poincaré inequality on $\bar{\Omega}$ with dilation $L^{\prime}$.

Recall that $\Omega$ is always $A$-quasiconvex by the $A$-uniformity condition, but that $L$ may be smaller than $A$. Also, $\bar{\Omega}$ is always $L$-quasiconvex, but it is possible to have $L^{\prime}<L$.

Proof Let $x_{0} \in \Omega, 0<r \leq 2 \operatorname{diam} \Omega$ and $B_{0}=B\left(x_{0}, r\right)$ be fixed. The balls in this proof are with respect to $\Omega$. It is well known, and easily shown using the arguments in the proof of Lemma 4.8, that uniform spaces satisfy the corkscrew condition, i.e. there exists $a_{0}$ (independent of $x_{0}$ and $r$ ) and $z$ such that $d_{\Omega}(z) \geq 2 a_{0} r$ and $B\left(z, a_{0} r\right) \subset B_{0}$, cf. Björn-Shanmugalingam [13, Lemma 4.2]. With $c_{0}$ as in the assumptions of the proposition, let

$$
r_{0}=\frac{a_{0} c_{0} r}{8 A} \leq \frac{c_{0} d_{\Omega}(z)}{16 A} \text { and } r_{i}=2^{-i} r_{0}, \quad i=1,2, \ldots
$$

Since $\Omega$ is $A$-uniform, [13, Lemma 4.3] with $\rho_{0}=r_{0}$ and $\sigma=1 / c_{0}$ provides us for every $x \in B_{0}$ with a chain

$$
\mathcal{B}_{x}=\left\{B_{i, j}=B_{\varepsilon}\left(x_{i, j}, r_{i}\right): i=0,1, \ldots \text { and } j=0,1, \ldots, m_{i}\right\}
$$

of balls connecting the ball $B_{0,0}:=B\left(z, r_{0}\right)$ to $x$ as follows:

(a) For all $i$ and $j$ we have $m_{i} \leq A r / r_{0}=8 A^{2} / a_{0} c_{0}$,

$$
4 r_{i} \leq c_{0} d_{\Omega}\left(x_{i, j}\right) \text { and } d\left(x_{i, j}, x\right) \leq 2^{-i} A d(x, z)<2^{-i} A r \text {. }
$$


(b) For large $i$, we have $m_{i}=0$ and the balls $B_{i, 0}$ are centered at $x$.

(c) The balls are ordered lexicographically, i.e. $B_{i, j}$ comes before $B_{i^{\prime}, j^{\prime}}$ if and only if $i<i^{\prime}$, or $i=i^{\prime}$ and $j<j^{\prime}$. If $B^{*}$ denotes the immediate successor of $B \in \mathcal{B}_{x}$ then $B \cap B^{*}$ is nonempty.

Let $u$ be a bounded measurable function on $\Omega$ and $g$ be an upper gradient of $u$ in $\Omega$. If $x \in B_{0}$ is a Lebesgue point of $u$ then

$$
\left|u(x)-u_{B_{0,0}}\right|=\lim _{i \rightarrow \infty}\left|u_{B_{i, 0}}-u_{B_{0,0}}\right| \leq \sum_{B \in \mathcal{B}_{x}}\left|u_{B^{*}}-u_{B}\right|,
$$

where $u_{B}=u_{B, v}$ and similarly for other balls. Moreover, $B^{*} \subset 3 B$ and

$$
\left|u_{B^{*}}-u_{B}\right| \leq\left|u_{B^{*}}-u_{3 B}\right|+\left|u_{3 B}-u_{B}\right|
$$

As $3 r_{i} \leq c_{0} d_{\Omega}\left(x_{i, j}\right)$ and the radii of $B$ and $B^{*}$ differ by at most a factor 2 , an application of the $p$-Poincaré inequality on $3 B$ shows that

$$
\left|u_{B^{*}}-u_{3 B}\right| \lesssim f_{3 B}\left|u-u_{3 B}\right| \mathrm{d} v \leq C r_{B}\left(f_{3 \lambda B} g^{p} \mathrm{~d} v\right)^{1 / p},
$$

where $r_{B}$ is the radius of $B$ and $\lambda$ is the dilation constant in the assumed $p$-Poincare inequality for subWhitney balls. The difference $\left|u_{3 B}-u_{B}\right|$ is estimated in the same way. Hence, inserting these estimates into (6.4),

$$
\left|u(x)-u_{B_{0,0}}\right| \lesssim \sum_{B \in \mathcal{B}_{x}} r_{B}\left(f_{3 \lambda B} g^{p} \mathrm{~d} \nu\right)^{1 / p} .
$$

We now wish to estimate the measure of level sets of the function $x \mapsto\left|u(x)-u_{B_{0,0}}\right|$ in $B_{0}$. Assume that $\left|u(x)-u_{B_{0,0}}\right| \geq t$ and write $t=C_{\alpha} N t \sum_{i=0}^{\infty} 2^{-i \alpha}$, where $\alpha \in(0,1)$ will be chosen later, and $N \leq 1+A r / R_{0}=1+8 A^{2} / a_{0} c_{0}$ is the maximal number of balls in $\mathcal{B}_{x}$ with the same radius. Then

$$
C_{\alpha} N t \sum_{i=0}^{\infty} 2^{-i \alpha}=t \lesssim \sum_{B \in \mathcal{B}_{x}} r_{B}\left(f_{3 \lambda B} g^{p} \mathrm{~d} v\right)^{1 / p}
$$

Hence, there exists $B_{x}=B\left(x_{i, j}, r_{i}\right) \in \mathcal{B}_{x}$ such that

$$
C_{\alpha} 2^{-i \alpha} t \lesssim r_{i}\left(f_{3 \lambda B_{x}} g^{p} \mathrm{~d} v\right)^{1 / p}
$$

We have $2^{-i}=r_{i} / r_{0}=8 A r_{i} / a_{0} c_{0} r$, and inserting this into the last inequality yields

$$
t \lesssim r\left(\frac{r_{i}}{r}\right)^{1-\alpha}\left(f_{3 \lambda B_{x}} g^{p} \mathrm{~d} \nu\right)^{1 / p}
$$


As $v$ is globally doubling, there exists $s>0$ independent of $B_{x}$ such that

$$
\frac{r_{i}}{r} \lesssim\left(\frac{v\left(3 \lambda B_{x}\right)}{\nu\left(B_{0}\right)}\right)^{1 / s}
$$

see e.g. [5, Lemma 3.3] or [30, (3.4.9)]. Hence

$$
t \lesssim r\left(\frac{v\left(3 \lambda B_{x}\right)}{v\left(B_{0}\right)}\right)^{(1-\alpha) / s}\left(f_{3 \lambda B_{x}} g^{p} \mathrm{~d} \nu\right)^{1 / p}
$$

and choosing $\alpha \in(0,1)$ so that $\theta:=1-(1-\alpha) p / s \in(0,1)$, we obtain

$$
\nu\left(3 \lambda B_{x}\right)^{\theta} \lesssim \frac{r^{p}}{t^{p} \nu\left(B_{0}\right)^{1-\theta}} \int_{3 \lambda B_{x}} g^{p} \mathrm{~d} \nu .
$$

Let $E_{t}=\left\{x \in B_{0}:\left|u(x)-u_{B_{0,0}}\right| \geq t\right\}$ and $F_{t}$ be the set of all points in $E_{t}$ which are Lebesgue points of $u$. The global doubling property of $v$ guarantees that a.e. $x$ is a Lebesgue point of $u$, see Heinonen [27, Theorem 1.8]. By the above, for every $x \in F_{t}$ there exists $B_{x} \in \mathcal{B}_{x}$ satisfying (6.5). Note also that by construction of the chain, we have $x \in B_{x}^{\prime}:=8\left(a_{0} c_{0}\right)^{-1} A^{2} B_{x}$. The balls $\left\{B_{x}^{\prime}\right\}_{x \in F_{t}}$, therefore cover $F_{t}$. The 5-covering lemma (Theorem 1.2 in Heinonen [27]) provides us with a pairwise disjoint collection $\left\{\lambda B_{x_{i}}^{\prime}\right\}_{i=1}^{\infty}$ such that the union of all balls $5 \lambda B_{x_{i}}^{\prime}$ covers $F_{t}$. Then the balls $3 \lambda B_{x_{i}} \subset \lambda B_{x_{i}}^{\prime}$ are also pairwise disjoint and the global doubling property of $v$, together with (6.5), yields

$$
\begin{aligned}
\nu\left(E_{t}\right)=v\left(F_{t}\right) & \leq \sum_{i=1}^{\infty} v\left(5 \lambda B_{x_{i}}^{\prime}\right) \lesssim \sum_{i=1}^{\infty} v\left(3 \lambda B_{x_{i}}\right) \\
& \lesssim \frac{r^{p / \theta}}{t^{p / \theta} v\left(B_{0}\right)^{1 / \theta-1}} \sum_{i=1}^{\infty}\left(\int_{3 \lambda B_{x_{i}}} g^{p} \mathrm{~d} v\right)^{1 / \theta} \\
& \leq \frac{r^{p / \theta}}{t^{p / \theta} v\left(B_{0}\right)^{1 / \theta-1}}\left(\int_{\Lambda B_{0}} g^{p} \mathrm{~d} v\right)^{1 / \theta}
\end{aligned}
$$

where $\Lambda$ depends only on $A, \lambda, a_{0}$ and $c_{0}$. Lemma 4.22 in Heinonen [27], which can be proved using the Cavalieri principle, now implies that

$$
f_{B_{0}}\left|u-u_{B_{0,0}}\right| \mathrm{d} v \lesssim r\left(f_{\Lambda B_{0}} g^{p} \mathrm{~d} v\right)^{1 / p}
$$

and a standard argument based on the triangle inequality allows us to replace $u_{B_{0,0}}$ by $u_{B_{0}}$.

Since $\Omega$ is $L$-quasiconvex, it follows from [5, Theorem 4.39] that the dilation $\Lambda$ in the obtained global $p$-Poincaré inequality can be replaced by $\mathrm{L}$. 
Finally, by Proposition 7.1 in Aikawa-Shanmugalingam [1] (or the proof above applied within $\bar{\Omega}$ and with $\left.x_{0} \in \bar{\Omega}\right)$, v supports a global $p$-Poincaré inequality, where, again using [5, Theorem 4.39], the dilation constant can be chosen to be $L^{\prime}$.

\section{Hyperbolization}

We assume in this section that $(\Omega, d)$ is a noncomplete L-quasiconvex space which is open in its completion $\bar{\Omega}$, and let $\partial \Omega$ be its boundary within $\bar{\Omega}$.

We define the quasihyperbolic metric on $\Omega$ by

$$
k(x, y)=\inf _{\gamma} \int_{\gamma} \frac{\mathrm{d} s}{d_{\Omega}(\gamma(s))}, \quad \text { where } d_{\Omega}(x)=\operatorname{dist}(x, \partial \Omega),
$$

$\mathrm{d} s$ is the arc length parametrization of $\gamma$, and the infimum is taken over all rectifiable curves in $\Omega$ connecting $x$ to $y$. It follows that $(\Omega, k)$ is a length space. Balls with respect to the quasihyperbolic metric $k$ will be denoted by $B_{k}$.

Even though our main interest is in hyperbolizing uniform spaces, the quasihyperbolic metric makes sense in greater generality. In fact, the results in this section hold also if we let $\Omega \varsubsetneqq Y$ be an $L$-quasiconvex open subset of a (not necessarily complete) metric space $Y$ and the quasihyperbolic metric $k$ is defined using $d_{\Omega}(x)=\operatorname{dist}(x, Y \backslash \Omega)$.

If $\Omega$ is a locally compact uniform space, then Theorem 3.6 in Bonk-HeinonenKoskela [14] shows that the space $(\Omega, k)$ is a proper geodesic Gromov hyperbolic space. Moreover, if $\Omega$ is bounded, then $(\Omega, k)$ is roughly starlike.

As described in the introduction, the operations of uniformization and hyperbolization are mutually opposite, by Bonk-Heinonen-Koskela [14, the discussion before Proposition 4.5].

Lemma 7.1 Let $x, y \in \Omega$. Then the following are true:

$$
\begin{aligned}
k(x, y) & \geq \frac{d(x, y)}{2 d_{\Omega}(x)}, & & \text { if } d(x, y) \leq d_{\Omega}(x), \\
k(x, y) & \geq \frac{1}{2}, & & \text { if } d(x, y) \geq d_{\Omega}(x), \\
\frac{d(x, y)}{2 d_{\Omega}(x)} \leq k(x, y) & \leq \frac{2 L d(x, y)}{d_{\Omega}(x)}, & & \text { if } d(x, y) \leq \frac{d_{\Omega}(x)}{2 L} .
\end{aligned}
$$

Moreover,

$$
\begin{array}{rlrl}
B\left(x, \frac{r d_{\Omega}(x)}{2 L}\right) \subset B_{k}(x, r) \subset B\left(x, 2 r d_{\Omega}(x)\right), & & \text { if } r \leq \frac{1}{2}, \\
B_{k}\left(x, \frac{r}{2 d_{\Omega}(x)}\right) \subset B(x, r) \subset B_{k}\left(x, \frac{2 L r}{d_{\Omega}(x)}\right), & \text { if } r \leq \frac{d_{\Omega}(x)}{2 L} .
\end{array}
$$

If $\Omega$ is $A$-uniform it is possible to get an upper bound similar to the one in (7.1) also when $d(x, y) \leq \frac{1}{2} d_{\Omega}(x)$, albeit with a little more complicated expression for the 
constant. As we will not need such an estimate, we leave it to the interested reader to deduce such a bound.

Proof Without loss of generality we assume that $x \neq y$.

Assume first that $d(x, y) \leq d_{\Omega}(x)$. Let $\gamma:\left[0, l_{\gamma}\right] \rightarrow \Omega$ be a curve from $x$ to $y$. All curves in this proof will be arc length parametrized rectifiable curves in $\Omega$. Then $l_{\gamma} \geq d(x, y)$ and

$$
\int_{\gamma} \frac{\mathrm{d} s}{d_{\Omega}(\gamma(s))} \geq \int_{0}^{d(x, y)} \frac{\mathrm{d} t}{d_{\Omega}(x)+t}>\int_{0}^{d(x, y)} \frac{\mathrm{d} t}{2 d_{\Omega}(x)}=\frac{d(x, y)}{2 d_{\Omega}(x)} .
$$

Taking infimum over all such $\gamma$ shows that $k(x, y) \geq d(x, y) / 2 d_{\Omega}(x)$.

Suppose next that $d(x, y) \geq d_{\Omega}(x)$. Let $\gamma:\left[0, l_{\gamma}\right] \rightarrow \Omega$ be a curve from $x$ to $y$. Then $l_{\gamma} \geq d(x, y) \geq d_{\Omega}(x)$ and

$$
\int_{\gamma} \frac{\mathrm{d} s}{d_{\Omega}(\gamma(s))} \geq \int_{0}^{d_{\Omega}(x)} \frac{\mathrm{d} t}{d_{\Omega}(x)+t}>\int_{0}^{d_{\Omega}(x)} \frac{\mathrm{d} t}{2 d_{\Omega}(x)}=\frac{1}{2} .
$$

Taking infimum over all such $\gamma$ shows that $k(x, y) \geq \frac{1}{2}$.

Assume finally that $d(x, y) \leq d_{\Omega}(x) / 2 L$. As $\Omega$ is $L$-quasiconvex, there is a curve $\gamma:\left[0, l_{\gamma}\right] \rightarrow \Omega$ from $x$ to $y$ with length $l_{\gamma} \leq L d(x, y) \leq \frac{1}{2} d_{\Omega}(x)$. Then

$$
k(x, y) \leq \int_{\gamma} \frac{\mathrm{d} s}{d_{\Omega}(\gamma(s))} \leq l_{\gamma} \frac{2}{d_{\Omega}(x)} \leq \frac{2 L d(x, y)}{d_{\Omega}(x)} .
$$

The ball inclusions now follow directly from this.

We shall now equip $(\Omega, k)$ with a measure determined by the original measure $\mu$ on $\Omega$. As before, for the results in this section it will be enough to assume that $\Omega$ is quasiconvex.

Definition 7.2 Let $\Omega$ be equipped with a Borel measure $\mu$. For measurable $A \subset \Omega$ and $\alpha>0$, let

$$
\mu^{\alpha}(A)=\int_{A} \frac{\mathrm{d} \mu(x)}{d_{\Omega}(x)^{\alpha}}
$$

Proposition 7.3 Assume that $\mu$ is globally doubling in $(\Omega, d)$ with doubling constant $C_{\mu}$. Then $\mu^{\alpha}$ is doubling for $B_{k}$-balls of radii at most $R_{0}=\frac{1}{8}$, with doubling constant $C_{d}=4{ }^{\alpha} C_{\mu}^{m}$, where $m=\left\lceil\log _{2} 8 L\right\rceil$.

Moreover, if $R_{1}>0$, then $\mu^{\alpha}$ is doubling for $B_{k}$-balls of radii at most $R_{1}$.

Proof Let $x \in \Omega, r \leq \frac{1}{8}, B_{k}=B_{k}(x, r)$ and $B=B\left(x, r d_{\Omega}(x)\right)$. By Lemma 7.1,

$$
\mu^{\alpha}\left(B_{k}\right) \geq \mu^{\alpha}\left(\frac{1}{2 L} B\right) \geq\left(\frac{1}{2 d_{\Omega}(x)}\right)^{\alpha} \mu\left(\frac{1}{2 L} B\right)
$$


and hence, again using Lemma 7.1,

$$
\begin{aligned}
\mu^{\alpha}\left(2 B_{k}\right) \leq \mu^{\alpha}(4 B) & \leq\left(\frac{2}{d_{\Omega}(x)}\right)^{\alpha} \mu(4 B) \leq\left(\frac{2}{d_{\Omega}(x)}\right)^{\alpha} C_{\mu}^{m} \mu\left(\frac{1}{2 L} B\right) \\
& \leq\left(\frac{2}{d_{\Omega}(x)}\right)^{\alpha}\left(2 d_{\Omega}(x)\right)^{\alpha} C_{\mu}^{m} \mu^{\alpha}\left(B_{k}\right)=C_{d} \mu^{\alpha}\left(B_{k}\right)
\end{aligned}
$$

As $(\Omega, k)$ is a length space, Lemma 3.5 shows that $\mu^{\alpha}$ is doubling for $B_{k}$-balls of radii at most $R_{1}$ for any $R_{1}>0$.

Proposition 7.4 Assume that $(\Omega, d)$ is equipped with a globally doubling measure $\mu$ supporting a global $p$-Poincaré inequality with dilation $\lambda$ and $p \geq 1$. Let $\alpha>0$ and $R_{1}>0$. Then $(\Omega, k)$, equipped with the measure $\mu^{\alpha}$, supports a p-Poincaré inequality for balls of radii at most $R_{1}$ with dilation $L$ and the other Poincaré constant depending only on $L, R_{1}$ and the global doubling and Poincaré constants.

Proof Let $u$ be a bounded measurable function on $\Omega$ and $\hat{g}$ be an upper gradient of $u$ with respect to $k$. Since the arc length parametrization $\mathrm{d} s_{k}$ with respect to $k$ satisfies

$$
\mathrm{d} s_{k}=\frac{\mathrm{d} s}{d_{\Omega}(\cdot)},
$$

we conclude that

$$
\int_{\gamma} \hat{g} \mathrm{~d} s_{k}=\int_{\gamma} \frac{\hat{g}}{d_{\Omega}(\cdot)} \mathrm{d} s
$$

and thus $g(z):=\hat{g}(z) / d_{\Omega}(z)$ is an upper gradient of $u$ with respect to $d$, see the proof of Lemma 6.1 for further details.

Next, let $x \in \Omega, 0<r \leq R_{0}:=1 / 8 \lambda L, B_{k}=B_{k}(x, r)$ and $B=B\left(x, 2 r d_{\Omega}(x)\right)$. We see, by Lemma 7.1, that

$$
\frac{1}{4 L} B \subset B_{k} \subset B \text { and } \lambda B \subset 4 \lambda L B_{k},
$$

where all the above balls have comparable $\mu$-measures, as well as comparable $\mu^{\alpha}$ measures. Note that $d_{\Omega}(z) \simeq d_{\Omega}(x)$ for all $z \in 4 \lambda L B_{k}$. Thus,

$$
\begin{aligned}
f_{B_{k}}\left|u-u_{B, \mu}\right| \mathrm{d} \mu^{\alpha} & \lesssim f_{B}\left|u-u_{B, \mu}\right| \mathrm{d} \mu \lesssim r d_{\Omega}(x)\left(f_{\lambda B} g^{p} \mathrm{~d} \mu\right)^{1 / p} \\
& \simeq r\left(f_{\lambda B} \hat{g}^{p} \mathrm{~d} \mu\right)^{1 / p} \lesssim r\left(f_{4 \lambda L B_{k}} \hat{g}^{p} \mathrm{~d} \mu^{\alpha}\right)^{1 / p} .
\end{aligned}
$$

A standard argument based on the triangle inequality makes it possible to replace $u_{B, \mu}$ on the left-hand side by $u_{B_{k}, \mu^{\alpha}}$, and thus $Y$ supports a $p$-Poincaré inequality for balls of radii $\leq R_{0}$, with dilation $4 \lambda L$. The conclusion now follows from Theorem 5.3. 
Remark 7.5 Let $X$ be a Gromov hyperbolic space, equipped with a measure $\mu$, and consider its uniformization $X_{\varepsilon}$, together with the measure $\mu_{\beta}, \beta>0$, as in Definition 4.1. With $\alpha=\beta / \varepsilon$, it is then easily verified that the pull-back to $X$ of the measure $\left(\mu_{\beta}\right)^{\alpha}$, defined on the hyperbolization $\left(X_{\varepsilon}, k\right)$ of $X_{\varepsilon}$, is comparable to the original measure $\mu$.

\section{An Indirect Product of Gromov Hyperbolic Spaces}

We assume in this section that $X$ and $Y$ are two locally compact roughly starlike Gromov $\delta$-hyperbolic spaces. We fix two points $z_{X} \in X$ and $z_{Y} \in Y$, and let $M$ be a common constant for the roughly starlike conditions with respect to $z_{X}$ and $z_{Y}$. We also assume that $0<\varepsilon \leq \varepsilon_{0}(\delta)$ and that $z_{X}$ and $z_{Y}$ serve as centers for the uniformizations $X_{\varepsilon}$ and $Y_{\varepsilon}$.

In general, the Cartesian product $X \times Y$ of two Gromov hyperbolic spaces $X$ and $Y$ need not be Gromov hyperbolic; for example, $\mathbf{R} \times \mathbf{R}$ is not Gromov hyperbolic. In this section, we shall construct an indirect product metric on $X \times Y$ that does give us a Gromov hyperbolic space, namely we set $X \times{ }_{\varepsilon} Y$ to be the Gromov hyperbolic space $\left(X_{\varepsilon} \times Y_{\varepsilon}, k\right)$. To do so, we first need to show that the Cartesian product of two uniform spaces, equipped with the sum of their metrics, is a uniform domain. This can be proved using Theorems 1 and 2 in Gehring-Osgood [22] together with Proposition 2.14 in Bonk-Heinonen-Koskela [14], but this would result in a highly nonoptimal uniformity constant. We instead give a more self-contained proof that also yields a better estimate of the uniformity constant for the Cartesian product.

Example 8.1 Recall that the uniformization $\mathbf{R}_{\varepsilon}$ of the hyperbolic 1-dimensional space $\mathbf{R}$ is isometric to $\left(-\frac{1}{\varepsilon}, \frac{1}{\varepsilon}\right)$, see Example 4.2. Hence, for all $\varepsilon>0, \mathbf{R}_{\varepsilon} \times \mathbf{R}_{\varepsilon}$ is a planar square region, which is biLipschitz equivalent to the planar disk. Thus also its hyperbolization $\mathbf{R} \times{ }_{\varepsilon} \mathbf{R}$ is biLipschitz equivalent to the hyperbolic disk, which is the model 2-dimensional hyperbolic space.

Lemma 8.2 Let $(\Omega, d)$ be a bounded A-uniform space. Then for every pair of points $x, y \in \Omega$ and for every $L$ with $d(x, y) \leq L \leq$ diam $\Omega$, there exists a curve $\gamma \subset \Omega$ of length

$$
\frac{L}{5 A} \leq l(\gamma) \leq(A+1) L
$$

connecting $x$ to $y$ and such that for all $z \in \gamma$,

$$
d_{\Omega}(z) \geq \frac{1}{16 A^{2}} \min \left\{l\left(\gamma_{x, z}\right), l\left(\gamma_{z, y}\right)\right\}
$$

where $\gamma_{x, z}$ and $\gamma_{z, y}$ are the subcurves of $\gamma$ from $x$ to $z$ and from $z$ to $y$, respectively.

Proof Choose $x_{0} \in \Omega$ such that $d_{\Omega}\left(x_{0}\right) \geq \frac{4}{5} \sup _{z \in \Omega} d_{\Omega}(z)$. Then for all $z \in \Omega$, with $\gamma_{z, x_{0}}$ being an $A$-uniform curve from $z$ to $x_{0}$, and $z^{\prime}$ its midpoint,

$$
d\left(z, x_{0}\right) \leq l\left(\gamma_{z, x_{0}}\right) \leq 2 A d_{\Omega}\left(z^{\prime}\right) \leq \frac{5}{2} A d_{\Omega}\left(x_{0}\right) .
$$


Hence diam $\Omega \leq 5 A d_{\Omega}\left(x_{0}\right)$. Now, let $x, y \in \Omega$ and $L$ be as in the statement of the lemma. Let $\gamma_{x, x_{0}}$ be an $A$-uniform curve from $x$ to $x_{0}$. We shall distinguish two cases:

1. If $L \leq 5 A l\left(\gamma_{x, x_{0}}\right)$ then let $\hat{\gamma}_{x}$ be the restriction of $\gamma$ to $[0, L / 10 A]$ and $\hat{x}=$ $\gamma(L / 10 A)$ be its new endpoint.

2. If $L \geq 5 A l\left(\gamma_{x, x_{0}}\right)$ then let $\gamma_{x}$ be the restriction of $\gamma$ to $\left[0, \frac{1}{2} l\left(\gamma_{x, x_{0}}\right)\right]$ and $\hat{x}=$ $\gamma\left(\frac{1}{2} l\left(\gamma_{x, x_{0}}\right)\right)$ be its new endpoint. Note that

$$
d_{\Omega}(\hat{x}) \geq d_{\Omega}\left(x_{0}\right)-\frac{l\left(\gamma_{x, x_{0}}\right)}{2} \geq \frac{\operatorname{diam} \Omega}{5 A}-\frac{L}{10 A} \geq \frac{L}{10 A} .
$$

Choose a curve $\gamma^{\prime}$ of length $L / 10 A$, which starts and ends at $\hat{x}$. Then for all $z \in \gamma^{\prime}$,

$$
d_{\Omega}(z) \geq d_{\Omega}(\hat{x})-\frac{L}{20 A} \geq \frac{L}{20 A} .
$$

Thus, concatenating $\gamma^{\prime}$ to $\gamma_{x}$ we obtain a curve $\hat{\gamma}_{x}$ from $x$ to $\hat{x}$ of length

$$
\frac{L}{10 A} \leq l\left(\hat{\gamma}_{x}\right) \leq \frac{L}{5 A}
$$

and such that for all $z \in \hat{\gamma}_{x}$,

$$
d_{\Omega}(z) \geq \frac{1}{\max \{4, A\}} l\left(\hat{\gamma}_{x, z}\right) \geq \frac{1}{4 A} l\left(\hat{\gamma}_{x, z}\right),
$$

where $\hat{\gamma}_{x, z}$ is the part of $\hat{\gamma}_{x}$ from $x$ to $z$. The curve $\hat{\gamma}_{x}$, obtained in case 1 , clearly satisfies (8.2) and (8.3) as well.

A similar construction, using an $A$-uniform curve from $y$ to $x_{0}$, provides us with a curve $\hat{\gamma}_{y}$ from $y$ to $\hat{y}$, satisfying (8.2) and (8.3) with $x$ replaced by $y$.

Now, let $\tilde{\gamma}$ be a uniform curve from $\hat{x}$ to $\hat{y}$ and let $\gamma$ be the concatenation of $\hat{\gamma}_{x}$ with $\tilde{\gamma}$ and $\hat{\gamma}_{y}$ (reversed). Since $d(\hat{x}, \hat{y}) \leq d(x, y)+2 L / 5 A \leq(1+2 / 5 A) L$, we see that

$$
l(\gamma) \leq A\left(1+\frac{2}{5 A}\right) L+\frac{2 L}{5 A} \leq(A+1) L
$$

and the right-hand side inequality in (8.1) holds, while the left-hand side follows from (8.2).

To prove the second property, in view of (8.3), it suffices to consider $z \in \tilde{\gamma}$. Without loss of generality, assume that the part $\tilde{\gamma}_{\hat{x}, z}$ of $\tilde{\gamma}$ from $\hat{x}$ to $z$ has length at most $\frac{1}{2} l(\tilde{\gamma})$. Note that (8.3), applied to the choice $z=\hat{x}$, gives

$$
d_{\Omega}(\hat{x}) \geq \frac{1}{4 A} l\left(\hat{\gamma}_{x}\right)
$$

Again, we distinguish two cases. 
1. If $\frac{1}{2} d_{\Omega}(\hat{x}) \geq l\left(\tilde{\gamma}_{\hat{x}, z}\right)$ then by $(8.4)$,

$$
d_{\Omega}(z) \geq d_{\Omega}(\hat{x})-l\left(\tilde{\gamma}_{\hat{x}, z}\right) \geq \frac{1}{2} d_{\Omega}(\hat{x}) \geq \max \left\{l\left(\tilde{\gamma}_{\hat{x}, z}\right), \frac{1}{8 A} l\left(\hat{\gamma}_{x}\right)\right\},
$$

and hence we obtain that

$$
d_{\Omega}(z) \geq \frac{1}{2} d_{\Omega}(\hat{x}) \geq \frac{1}{16 A}\left(l\left(\tilde{\gamma}_{\hat{x}, z}\right)+l\left(\hat{\gamma}_{x}\right)\right)=\frac{1}{16 A} l\left(\gamma_{x, z}\right),
$$

where $\gamma_{x, z}$ is the part of $\gamma$ from $x$ to $z$.

2. On the other hand, if $\frac{1}{2} d_{\Omega}(\hat{x}) \leq l\left(\tilde{\gamma}_{\hat{x}, z}\right)$ then by (8.4) again,

$$
d_{\Omega}(z) \geq \frac{1}{A} l\left(\tilde{\gamma}_{\hat{x}, z}\right) \geq \frac{1}{2 A} d_{\Omega}(\hat{x}) \geq \frac{1}{8 A^{2}} l\left(\hat{\gamma}_{x}\right) .
$$

We conclude that

$$
d_{\Omega}(z) \geq \frac{1}{16 A^{2}}\left(l\left(\tilde{\gamma}_{\hat{x}, z}\right)+l\left(\hat{\gamma}_{x}\right)\right)=\frac{1}{16 A^{2}} l\left(\gamma_{x, z}\right)
$$

Proposition 8.3 Let $(\Omega, d)$ and $\left(\Omega^{\prime}, d^{\prime}\right)$ be two bounded uniform spaces, with diameters and uniformity constants $D, D^{\prime}, A$ and $A^{\prime}$, respectively. Then $\widetilde{\Omega}=\Omega \times \Omega^{\prime}$ is also a bounded uniform space with respect to the metric

$$
\tilde{d}\left(\left(x, x^{\prime}\right),\left(y, y^{\prime}\right)\right)=d(x, y)+d^{\prime}\left(x^{\prime}, y^{\prime}\right),
$$

with uniformity constant

$$
\tilde{A}=\frac{80\left[(A+1) D+\left(A^{\prime}+1\right) D^{\prime}\right]}{\min \left\{D / A^{3}, D^{\prime} /\left(A^{\prime}\right)^{3}\right\}} .
$$

Proof The boundedness is clear. Let $\tilde{x}=\left(x, x^{\prime}\right)$ and $\tilde{y}=\left(y, y^{\prime}\right)$ be two distinct points in $\widetilde{\Omega}$, and let

$$
\begin{aligned}
\Lambda & =\max \left\{\frac{d(x, y)}{D}, \frac{d^{\prime}\left(x^{\prime}, y^{\prime}\right)}{D^{\prime}}\right\} \leq 1, \\
L & =\Lambda D \geq d(x, y), \\
L^{\prime} & =\Lambda D^{\prime} \geq d^{\prime}\left(x^{\prime}, y^{\prime}\right) .
\end{aligned}
$$

Note that

$$
\Lambda\left(D+D^{\prime}\right) \geq \tilde{d}(\tilde{x}, \tilde{y}) \geq \Lambda \min \left\{D, D^{\prime}\right\} \geq \Lambda \min \left\{\frac{D}{A^{3}}, \frac{D^{\prime}}{\left(A^{\prime}\right)^{3}}\right\}
$$


We use Lemma 8.2 to find curves $\gamma \subset \Omega$ and $\gamma^{\prime} \subset \Omega^{\prime}$, connecting $x$ to $y$ and $x^{\prime}$ to $y^{\prime}$, respectively, of lengths

$$
\frac{L}{5 A} \leq l(\gamma) \leq(A+1) L \text { and } \frac{L^{\prime}}{5 A^{\prime}} \leq l\left(\gamma^{\prime}\right) \leq\left(A^{\prime}+1\right) L^{\prime},
$$

and such that for all $z \in \gamma$,

$$
d_{\Omega}(z) \geq \frac{1}{16 A^{2}} \min \left\{l\left(\gamma_{x, z}\right), l\left(\gamma_{z, y}\right)\right\}
$$

where $\gamma_{x, z}$ and $\gamma_{z, y}$ are the parts of $\gamma$ from $x$ to $z$ and from $z$ to $y$, respectively; similar statements holding true for $z^{\prime} \in \gamma^{\prime}$ and $A^{\prime}$. Note that $\Lambda>0$ since $\tilde{x} \neq \tilde{y}$. Hence $L, L^{\prime}>0$ and, by (8.7), the curves $\gamma$ and $\gamma^{\prime}$ are nonconstant.

Next, assuming that $\gamma$ and $\gamma^{\prime}$ are arc length parametrized, we show that the curve

$$
\tilde{\gamma}(t)=\left(\gamma\left(\frac{t}{l(\gamma)}\right), \gamma^{\prime}\left(\frac{t}{l\left(\gamma^{\prime}\right)}\right)\right), \quad t \in[0,1],
$$

is an $\tilde{A}$-uniform curve in $\widetilde{\Omega}$ connecting $\tilde{x}$ to $\tilde{y}$. To see this, note that we have by the definition (8.5) of $\tilde{d}$ that for all $0 \leq s \leq t \leq 1$, using (8.7) and then (8.6),

$$
\begin{aligned}
l\left(\left.\tilde{\gamma}\right|_{[s, t]}\right)=(t-s)\left(l(\gamma)+l\left(\gamma^{\prime}\right)\right) & \leq(t-s)\left[(A+1) D+\left(A^{\prime}+1\right) D^{\prime}\right] \Lambda \\
& \leq(t-s) \tilde{A} \tilde{d}(\tilde{x}, \tilde{y}) .
\end{aligned}
$$

In particular, $\tilde{\gamma}$ has the correct length. Since

$$
\partial \widetilde{\Omega}=\left(\partial \Omega \times \Omega^{\prime}\right) \cup\left(\Omega \times \partial \Omega^{\prime}\right) \cup\left(\partial \Omega \times \partial \Omega^{\prime}\right),
$$

we see that for all $\tilde{\gamma}(t)=\left(z, z^{\prime}\right)$ with $0 \leq t \leq \frac{1}{2}$, using (8.8) and then (8.7),

$$
d_{\Omega}(z) \geq \frac{l(\gamma) t}{16 A^{2}} \geq \frac{L t}{80 A^{3}}=\frac{\Lambda D t}{80 A^{3}}
$$

and similarly $d_{\Omega}\left(z^{\prime}\right) \geq \Lambda D^{\prime} t / 80\left(A^{\prime}\right)^{3}$. Thus, using (8.7) for the last inequality,

$$
\begin{aligned}
d_{\widetilde{\Omega}}(\tilde{\gamma}(t)) & =\min \left\{d_{\Omega}(z), d_{\Omega^{\prime}}\left(z^{\prime}\right)\right\} \geq \frac{\Lambda t}{80} \min \left\{\frac{D}{A^{3}}, \frac{D^{\prime}}{\left(A^{\prime}\right)^{3}}\right\} \\
& =\frac{t}{\tilde{A}}\left[(A+1) L+\left(A^{\prime}+1\right) L^{\prime}\right] \geq \frac{t}{\tilde{A}}\left[l(\gamma)+l\left(\gamma^{\prime}\right)\right]=\frac{l\left(\tilde{\gamma}_{\tilde{x}, \tilde{\gamma}(t)}\right)}{\tilde{A}}
\end{aligned}
$$

As a similar estimate holds for $\frac{1}{2} \leq t \leq 1$, we see that $\tilde{\gamma}$ is indeed an $\tilde{A}$-uniform curve.

We next see that the projection map $\pi: X \times_{\varepsilon} Y \rightarrow X$ given by $\pi((x, y))=x$ is Lipschitz continuous. 
Proposition 8.4 The above-defined projection map $\pi: X \times_{\varepsilon} Y \rightarrow X$ is $(C / \varepsilon)$ Lipschitz continuous, with $C$ depending only on $\varepsilon_{0}$ and $M$.

Proof Since $X \times{ }_{\varepsilon} Y$ is geodesic, it suffices to show that $\pi$ is locally $(C / \varepsilon)$-Lipschitz with $C$ independent of the locality. With $C_{1}=e^{-(1+\varepsilon M)}$ and $C_{2}=2 e\left(2 e^{\varepsilon M}-1\right)$ as in Theorem 2.10, for $(x, y) \in X \times Y$ let

$$
r=\frac{C_{1} \min \left\{d_{\varepsilon}(x), d_{\varepsilon}(y)\right\}}{2 C_{2}}, \quad x^{\prime} \in B_{\varepsilon}(x, r) \quad \text { and } \quad y^{\prime} \in B_{\varepsilon}(y, r) .
$$

The last part of Theorem 2.10 together with Lemma 2.8 then gives

$$
d\left(\pi(x, y), \pi\left(x^{\prime}, y^{\prime}\right)\right)=d\left(x, x^{\prime}\right) \simeq \frac{d_{\varepsilon}\left(x, x^{\prime}\right)}{\rho_{\varepsilon}(x)} \simeq \frac{d_{\varepsilon}\left(x, x^{\prime}\right)}{\varepsilon d_{\varepsilon}(x)},
$$

with comparison constants depending only on $\varepsilon_{0}$ and $M$.

Let $k_{\varepsilon}$ denote the quasihyperbolic metric on $\Omega:=X_{\varepsilon} \times Y_{\varepsilon}$. Note that since both $X_{\varepsilon}$ and $Y_{\varepsilon}$ are length spaces, so is $\Omega$. As $C_{2} / C_{1}>2 e$, we see that

$$
d_{\Omega}((x, y))=\min \left\{d_{\varepsilon}(x), d_{\varepsilon}(y)\right\}>2 e\left(d_{\varepsilon}\left(x, x^{\prime}\right)+d_{\varepsilon}\left(y, y^{\prime}\right)\right)
$$

and thus (7.1) in Lemma 7.1 with $L=e$ yields

$$
k_{\varepsilon}\left((x, y),\left(x^{\prime}, y^{\prime}\right)\right) \simeq \frac{d_{\varepsilon}\left(x, x^{\prime}\right)+d_{\varepsilon}\left(y, y^{\prime}\right)}{\min \left\{d_{\varepsilon}(x), d_{\varepsilon}(y)\right\}} .
$$

It follows that

$$
d\left(\pi(x, y), \pi\left(x^{\prime}, y^{\prime}\right)\right) \lesssim \frac{1}{\varepsilon} k_{\varepsilon}\left((x, y),\left(x^{\prime}, y^{\prime}\right)\right) .
$$

Next, we shall see how $X \times_{\varepsilon} Y$ compares to $X \times{ }_{\varepsilon^{\prime}} Y$.

Proposition 8.5 Let $0<\varepsilon^{\prime}<\varepsilon \leq \varepsilon_{0}(\delta)$. The canonical identity maps

$$
\Phi: X \times{ }_{\varepsilon} Y \rightarrow X \times{ }_{\varepsilon^{\prime}} Y \text { and } \Psi: X_{\varepsilon^{\prime}} \times Y_{\varepsilon^{\prime}} \rightarrow X_{\varepsilon} \times Y_{\varepsilon}
$$

are Lipschitz continuous. More precisely, there is a constant $C^{\prime}$, depending only on $\varepsilon_{0}$ and $M$, such that $\Phi$ is $\left(C^{\prime} \varepsilon^{\prime} / \varepsilon\right)$-Lipschitz while $\Psi$ is $C^{\prime}$-Lipschitz.

Moreover, neither $\Phi^{-1}$ nor $\Psi^{-1}$ is Lipschitz continuous.

Proof We first consider $\Phi$. Since $X \times_{\varepsilon} Y$ is geodesic, it suffices to show that $\Phi$ is locally $\left(C^{\prime} \varepsilon^{\prime} / \varepsilon\right)$-Lipschitz with $C^{\prime}$ independent of the locality. As in the proof of Proposition 8.4, for $(x, y) \in X \times Y$ and $C_{1}, C_{2}$ from Theorem 2.10, let

$$
r=\frac{C_{1} \min \left\{d_{\varepsilon}(x), d_{\varepsilon^{\prime}}(x), d_{\varepsilon}(y), d_{\varepsilon^{\prime}}(y)\right\}}{2 C_{2}}, \quad x^{\prime} \in B_{\varepsilon}(x, r) \quad \text { and } \quad y^{\prime} \in B_{\varepsilon}(y, r) .
$$


Theorem 2.10 then gives

$$
d_{\varepsilon}\left(x, x^{\prime}\right) \simeq \rho_{\varepsilon}(x) d\left(x, x^{\prime}\right) \text { and } d_{\varepsilon}\left(y, y^{\prime}\right) \simeq \rho_{\varepsilon}(y) d\left(y, y^{\prime}\right)
$$

Let $\tilde{d}_{\varepsilon}, \tilde{d}_{\mathcal{\varepsilon}^{\prime}}, k_{\varepsilon}$ and $k_{\mathcal{E}^{\prime}}$ denote the product metrics as in (8.5) and the quasihyperbolic metrics on $X_{\varepsilon} \times Y_{\varepsilon}$ and $X_{\mathcal{\varepsilon}^{\prime}} \times Y_{\mathcal{\varepsilon}^{\prime}}$ respectively. As in (8.9), we conclude that

$$
k_{\varepsilon}\left((x, y),\left(x^{\prime}, y^{\prime}\right)\right) \simeq \frac{d_{\varepsilon}\left(x, x^{\prime}\right)+d_{\varepsilon}\left(y, y^{\prime}\right)}{\min \left\{d_{\varepsilon}(x), d_{\varepsilon}(y)\right\}} .
$$

Without loss of generality we assume that $\rho_{\varepsilon}(x) \leq \rho_{\varepsilon}(y)$, and then using Lemma 2.8,

$$
d_{\varepsilon}(x) \simeq \frac{\rho_{\varepsilon}(x)}{\varepsilon} \leq \frac{\rho_{\varepsilon}(y)}{\varepsilon} \simeq d_{\varepsilon}(y)
$$

in which case we also have that

$$
d_{\mathcal{E}^{\prime}}(x) \simeq \frac{\rho_{\varepsilon^{\prime}}(x)}{\varepsilon^{\prime}} \leq \frac{\rho_{\varepsilon^{\prime}}(y)}{\varepsilon^{\prime}} \simeq d_{\mathcal{E}^{\prime}}(y)
$$

Therefore, using (8.10),

$$
k_{\varepsilon}\left((x, y),\left(x^{\prime}, y^{\prime}\right)\right) \simeq \frac{d_{\varepsilon}\left(x, x^{\prime}\right)+d_{\varepsilon}\left(y, y^{\prime}\right)}{d_{\varepsilon}(x)} \simeq \varepsilon\left(d\left(x, x^{\prime}\right)+\frac{\rho_{\varepsilon}(y)}{\rho_{\varepsilon}(x)} d\left(y, y^{\prime}\right)\right),
$$

with a similar statement holding true for $\varepsilon^{\prime}$. Since

$$
\frac{\rho_{\varepsilon^{\prime}}(y)}{\rho_{\varepsilon^{\prime}}(x)}=\left(\frac{\rho_{\varepsilon}(y)}{\rho_{\varepsilon}(x)}\right)^{\varepsilon^{\prime} / \varepsilon} \leq \frac{\rho_{\varepsilon}(y)}{\rho_{\varepsilon}(x)}
$$

we conclude from (8.11) that

$$
k_{\mathcal{\varepsilon}^{\prime}}\left((x, y),\left(x^{\prime}, y^{\prime}\right)\right) \lesssim \frac{\varepsilon^{\prime}}{\varepsilon} k_{\varepsilon}\left((x, y),\left(x^{\prime}, y^{\prime}\right)\right),
$$

which proves the Lipschitz continuity of $\Phi$.

We now compare the product uniform domains $X_{\varepsilon} \times Y_{\varepsilon}$ and $X_{\mathcal{\varepsilon}^{\prime}} \times Y_{\mathcal{\varepsilon}^{\prime}}$. With $(x, y),\left(x^{\prime}, y^{\prime}\right) \in X \times Y$ as in the first part of the proof, we have by (8.10) and the assumption $0<\varepsilon^{\prime}<\varepsilon$ that

$$
\begin{aligned}
\tilde{d}_{\varepsilon}\left((x, y),\left(x^{\prime}, y^{\prime}\right)\right) & =d_{\varepsilon}\left(x, x^{\prime}\right)+d_{\varepsilon}\left(y, y^{\prime}\right) \\
& \simeq \rho_{\varepsilon}(x) d\left(x, x^{\prime}\right)+\rho_{\varepsilon}(y) d\left(y, y^{\prime}\right) \\
& \leq \rho_{\varepsilon^{\prime}}(x) d\left(x, x^{\prime}\right)+\rho_{\varepsilon^{\prime}}(y) d\left(y, y^{\prime}\right) \\
& \simeq \tilde{d}_{\varepsilon^{\prime}}\left((x, y),\left(x^{\prime}, y^{\prime}\right)\right),
\end{aligned}
$$


which proves the Lipschitz continuity of $\Psi$. On the other hand, choosing $y=y^{\prime}=z_{Y}$, with $\rho_{\varepsilon}\left(z_{Y}\right)=1$, gives

$$
\frac{\tilde{d}_{\varepsilon^{\prime}}\left(\left(x, z_{Y}\right),\left(x^{\prime}, z_{Y}\right)\right)}{\tilde{d}_{\varepsilon}\left(\left(x, z_{Y}\right),\left(x^{\prime}, z_{Y}\right)\right)} \simeq \frac{\rho_{\varepsilon^{\prime}}(x)}{\rho_{\varepsilon}(x)}=\rho_{\varepsilon}(x)^{-1+\varepsilon^{\prime} / \varepsilon} .
$$

Since $\varepsilon^{\prime}<\varepsilon$, letting $d\left(x, z_{X}\right) \rightarrow \infty$ and so $\rho_{\varepsilon}(x) \rightarrow 0$ shows that $\Psi^{-1}$ is not Lipschitz.

To show that $\Phi^{-1}$ is not Lipschitz, let $x_{j} \in X$ be such that $\rho_{\varepsilon}\left(x_{j}\right) \rightarrow 0$ (and equivalently, $\rho_{\varepsilon^{\prime}}\left(x_{j}\right) \rightarrow 0$ ) as $j \rightarrow \infty$. With $C(\delta)$ as in (2.2) and $C_{1}, C_{2}$ as in Theorem 2.10, for $j=1,2, \ldots$ we choose $y_{j} \in Y$ such that

$$
d\left(z_{Y}, y_{j}\right)=\frac{C_{1} d_{\varepsilon}\left(x_{j}\right)}{4 C_{2} C(\delta)}
$$

This is possible since $Y$ is geodesic. Then, for sufficiently large $j$, we have $\varepsilon d\left(z_{Y}, y_{j}\right) \leq 1$ and hence by $(2.2)$,

$$
d_{\varepsilon}\left(z_{Y}, y_{j}\right) \leq C(\delta) d\left(z_{Y}, y_{j}\right)=\frac{C_{1} d_{\varepsilon}\left(x_{j}\right)}{4 C_{2}}
$$

Since also $\rho_{\varepsilon}\left(x_{j}\right) \leq 1=\rho_{\varepsilon}\left(z_{Y}\right)$, we thus conclude from (8.11), with the choice $x=x^{\prime}=x_{j}, y=z_{Y}$ and $y^{\prime}=y_{j}$, that

$$
k_{\varepsilon}\left(\left(x_{j}, z_{Y}\right),\left(x_{j}, y_{j}\right)\right) \simeq \frac{\varepsilon d\left(z_{Y}, y_{j}\right)}{\rho_{\varepsilon}\left(x_{j}\right)}
$$

with a similar statement holding also for $\varepsilon^{\prime}$. This shows that

$$
\frac{k_{\varepsilon}\left(\left(x_{j}, z_{Y}\right),\left(x_{j}, y_{j}\right)\right)}{k_{\varepsilon^{\prime}}\left(\left(x_{j}, z_{Y}\right),\left(x_{j}, y_{j}\right)\right)} \simeq \frac{\varepsilon \rho_{\varepsilon^{\prime}}\left(x_{j}\right)}{\varepsilon^{\prime} \rho_{\varepsilon}\left(x_{j}\right)}=\frac{\varepsilon}{\varepsilon^{\prime}} \rho_{\varepsilon}\left(x_{j}\right)^{-1+\varepsilon^{\prime} / \varepsilon} \rightarrow \infty, \quad \text { as } j \rightarrow \infty \text {. }
$$

i.e. $\Phi^{-1}$ is not Lipschitz.

Remark 8.6 If $X=Y=\mathbf{R}$ then, according to Example 8.1, all the indirect products $\mathbf{R} \times{ }_{\varepsilon} \mathbf{R}$ are mutually biLipschitz equivalent. However, Proposition 8.5 shows that this equivalence cannot be achieved by the canonical identity map $\Phi$.

By Theorem 1.1 in Bonk-Heinonen-Koskela [14], $\Phi$ is biLipschitz if and only if $\Psi$ is a quasisimilarity. Note that $X_{\varepsilon}$ and $X_{\varepsilon^{\prime}}$ are quasisymmetrically equivalent by [14], and so are $Y_{\varepsilon}$ and $Y_{\varepsilon^{\prime}}$. On the other hand, products of quasisymmetric maps need not be quasisymmetric, as exhibited by the Rickman rug $\left([0,1], d_{\text {Euc }}\right) \times\left([0,1], d_{\text {Euc }}^{\alpha}\right)$ for $0<\alpha<1$, see Bishop-Tyson [2, Remark 1, Sect. 5] and DiMarco [21, Sect. 1]. This seems to happen whenever one of the component spaces has dimension 1 and the other has dimension larger than 1. 
Example 8.7 Let $X$ be the unit disk in $\mathbf{R}^{2}$, equipped with the Poincaré metric $k$, making it a Gromov hyperbolic space. Let $Y=(-1,1)$ be equipped with the quasihyperbolic metric (and so it is isometric to $\mathbf{R}$, see Examples 4.2 and 4.3). For both $X$ and $Y$ we can choose $\varepsilon=1$, resulting in $X_{1}$ being the Euclidean unit disk and $Y_{1}$ being the Euclidean interval $(-1,1)$. Thus $X_{1} \times Y_{1}$ is a solid 3-dimensional Euclidean cylinder, with boundary made up of $\mathbf{S}^{1} \times[-1,1]$ together with two copies of the disk.

Choosing $0<\varepsilon<1$, we instead obtain $X_{\varepsilon}$ and $Y_{\varepsilon}$, with $Y_{\varepsilon}$ isometric to the Euclidean interval $(-1 / \varepsilon, 1 / \varepsilon)$, see Example 4.2. The boundary of $X_{\varepsilon} \times Y_{\varepsilon}$ is made up of two copies of $X_{\varepsilon}$ together with $Z \times[-1 / \varepsilon, 1 / \varepsilon]$, where $Z$ is the $\varepsilon$-snowflaking of $\mathbf{S}^{1}$, which results in $Z$ being biLipschitz equivalent to a generalized von Koch snowflake loop.

If $X \times_{\varepsilon} Y$ were biLipschitz equivalent to $X \times_{1} Y$, then $Z \times[-1 / \varepsilon, 1 / \varepsilon]$ would be quasisymmetrically equivalent to a 2-dimensional region in $\partial\left(X_{1} \times Y_{1}\right)$, which is impossible as pointed out before this example.

\section{Newtonian Spaces and $p$-Harmonic Functions}

We assume in this section that $1 \leq p<\infty$ and that $Y=(Y, d, v)$ is a metric space equipped with a complete Borel measure v such that $0<v(B)<\infty$ for all balls $B \subset Y$.

For proofs of the facts stated in this section we refer the reader to Björn-Björn [5] and Heinonen-Koskela-Shanmugalingam-Tyson [30].

Following Shanmugalingam [36], we define a version of Sobolev spaces on $Y$.

Definition 9.1 For a measurable function $u: Y \rightarrow[-\infty, \infty]$, let

$$
\|u\|_{N^{1, p}(Y)}=\left(\int_{Y}|u|^{p} \mathrm{~d} \nu+\inf _{g} \int_{Y} g^{p} \mathrm{~d} \nu\right)^{1 / p},
$$

where the infimum is taken over all upper gradients $g$ of $u$. The Newtonian space on $Y$ is

$$
N^{1, p}(Y)=\left\{u:\|u\|_{N^{1, p}(Y)}<\infty\right\} .
$$

In this paper we assume that functions in $N^{1, p}(Y)$ are defined everywhere (with values in $[-\infty, \infty]$ ), not just up to an equivalence class in the corresponding function space. This is important in Definition 5.1, to make sense of $g$ being an upper gradient of $u$. The space $N^{1, p}(Y) / \sim$, where $u \sim v$ if and only if $\|u-v\|_{N^{1, p}(Y)}=0$, is a Banach space and a lattice. For a measurable set $E \subset Y$, the Newtonian space $N^{1, p}(E)$ is defined by considering $\left(E,\left.d\right|_{E},\left.v\right|_{E}\right)$ as a metric space in its own right. We say that $f \in N_{\text {loc }}^{1, p}(\Omega)$, where $\Omega$ is an open subset of $X$, if for every $x \in \Omega$ there exists $r_{x}>0$ such that $B\left(x, r_{x}\right) \subset \Omega$ and $f \in N^{1, p}\left(B\left(x, r_{x}\right)\right)$. The space $L_{\text {loc }}^{p}(\Omega)$ is defined similarly. 
Definition 9.2 The (Sobolev) capacity of a set $E \subset Y$ is the number

$$
C_{p}(E):=C_{p}^{Y}(E):=\inf _{u}\|u\|_{N^{1, p}(Y)}^{p},
$$

where the infimum is taken over all $u \in N^{1, p}(Y)$ such that $u=1$ on $E$.

A property is said to hold quasieverywhere (q.e.) if the set of all points at which the property fails has $C_{p}$-capacity zero. The capacity is the correct gauge for distinguishing between two Newtonian functions. If $u \in N^{1, p}(Y)$, then $u \sim v$ if and only if $u=v$ q.e. Moreover, if $u, v \in N_{\mathrm{loc}}^{1, p}(Y)$ and $u=v$ a.e., then $u=v$ q.e.

We will also need the variational capacity.

Definition 9.3 Let $\Omega \subset Y$ be open. Then

$$
N_{0}^{1, p}(\Omega):=\left\{\left.u\right|_{\Omega}: u \in N^{1, p}(Y) \text { and } u=0 \text { on } Y \backslash \Omega\right\}
$$

The variational capacity of $E \subset \Omega$ with respect to $\Omega$ is

$$
\operatorname{cap}_{p}(E, \Omega):=\operatorname{cap}_{p}^{Y}(E, \Omega):=\inf _{u} \int_{\Omega} g_{u}^{p} \mathrm{~d} \nu
$$

where the infimum is taken over all $u \in N_{0}^{1, p}(\Omega)$ such that $u=1$ on $E$.

The following lemma provides us with a sufficient condition for when a set has positive capacity, in terms of Hausdorff measures. It is similar to Proposition 4.3 in Lehrbäck [35], but the dimension condition for $s$ is weaker here and is only required for $x \in E$. For the reader's convenience, we provide a complete proof. We will use Lemma 9.4 to deduce Proposition 10.10.

Lemma 9.4 Let $(Y, d, v)$ be a complete metric space equipped with a globally doubling measure $v$ supporting a global p-Poincaré inequality. Let $E \subset Y$ be a Borel set of positive $\kappa$-dimensional Hausdorff measure and assume that for some $C, s, r_{0}>0$,

$$
v(B(x, r)) \geq C r^{s} \text { for all } x \in E \text { and all } 0<r \leq r_{0} .
$$

Then $C_{p}^{Y}(E)>0$ whenever $p>s-\kappa$.

Note that if (9.1) holds for some $r_{0}$, then it holds with $r_{0}=1$, although $C$ may change.

Proof By the regularity of the Hausdorff measure, there is a compact set $K \subset E$ with positive $\kappa$-dimensional Hausdorff measure. Assume that $C_{p}^{Y}(K)=0$. Then also the variational capacity $\operatorname{cap}_{p}^{Y}(K, B)=0$ for every ball $B \supset K$. By splitting $K$ into finitely many pieces if necessary, and shrinking $B$, we can assume that $\nu(2 B \backslash B)>0$.

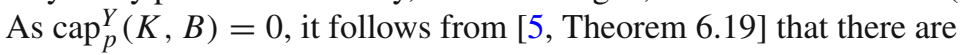

$$
u_{k} \in \operatorname{Lip}_{0}(B):=\{\varphi \in \operatorname{Lip}(Y): \varphi=0 \text { in } Y \backslash B\}
$$


with upper gradients $g_{k}$ such that $u_{k}=1$ on $K, 0 \leq u_{k} \leq 1$ on $Y$ and

$$
\int_{Y} g_{k}^{p} \mathrm{~d} v \rightarrow 0 \text { as } k \rightarrow \infty
$$

We can assume that $r_{0} \leq \operatorname{dist}(K, Y \backslash B)$ and set $r_{j}=2^{-j} r_{0}, j=0,1, \ldots$ For a fixed $x \in K$, consider the balls $B_{j}=B\left(x, r_{j}\right)$. A standard telescoping argument, using the doubling property of $v$ together with the $p$-Poincare inequality, then shows that for a fixed $k$ and $u:=u_{k}$,

$$
\begin{aligned}
\left|u(x)-u_{B_{0}}\right| & \leq \sum_{j=0}^{\infty}\left|u_{B_{j+1}}-u_{B_{j}}\right| \lesssim \sum_{j=0}^{\infty} f_{B_{j}}\left|u-u_{B_{j}}\right| \mathrm{d} \nu \\
& \lesssim \sum_{j=0}^{\infty} \frac{r_{j}}{v\left(B_{j}\right)^{1 / p}}\left(\int_{\lambda B_{j}} g_{k}^{p} \mathrm{~d} v\right)^{1 / p} .
\end{aligned}
$$

Because $u$ vanishes outside $B$ and $v(2 B \backslash B)>0$, we see that

$$
u_{2 B}:=f_{2 B} u \mathrm{~d} v \leq \frac{v(B)}{v(2 B)}=: 1-2 \theta<1 .
$$

Moreover,

$$
\left|u_{2 B}-u_{B_{0}}\right| \lesssim \frac{r_{B}}{v(2 \lambda B)^{1 / p}}\left(\int_{2 \lambda B} g_{k}^{p} \mathrm{~d} \nu\right)^{1 / p} \rightarrow 0, \quad \text { as } k \rightarrow \infty,
$$

where $r_{B}$ stands for the radius of $B$. Since $u(x)=1$, we conclude that for sufficiently large $k$, independently of $x \in K$,

$$
\left|u(x)-u_{B_{0}}\right| \geq\left|u(x)-u_{2 B}\right|-\left|u_{2 B}-u_{B_{0}}\right| \geq 2 \theta-\left|u_{2 B}-u_{B_{0}}\right| \geq \theta \simeq \sum_{j=0}^{\infty} r_{j}^{\tau},
$$

where $\tau=1-(s-\kappa) / p>0$. Inserting this into (9.2) and comparing the sums, we see that for each $x \in K$ there exists a ball $B_{x}=B_{j(x)}$ centered at $x$ and with radius $r_{x}=r_{j(x)}$ such that

$$
\int_{\lambda B_{x}} g_{k}^{p} \mathrm{~d} \nu \gtrsim \frac{v\left(B_{x}\right)}{r_{x}^{p(1-\tau)}} \gtrsim r_{x}^{\kappa}
$$

because of the assumption (9.1). Using the 5-covering lemma, we can out of the balls $\lambda B_{x}$ choose a countable pairwise disjoint subcollection $\lambda \widehat{B}_{j}, j=1,2, \ldots$, with radii $\hat{r}_{j}$, so that $K \subset \bigcup_{j=1}^{\infty} 5 \lambda \widehat{B}_{j}$. Hence using (9.3) we obtain

$$
\sum_{j=1}^{\infty} \hat{r}_{j}^{\kappa} \lesssim \sum_{j=1}^{\infty} \int_{\lambda \widehat{B}_{j}} g_{k}^{p} \mathrm{~d} v \lesssim \int_{B} g_{k}^{p} \mathrm{~d} v \rightarrow 0, \quad \text { as } k \rightarrow \infty
$$


showing that the $\kappa$-dimensional Hausdorff content (and thus also the corresponding measure) is zero. This causes a contradiction, which concludes the proof.

Definition 9.5 Assume that $Y$ is complete. Let $\Omega \subset Y$ be open. Then $u \in N_{\text {loc }}^{1, p}(\Omega)$ is p-harmonic in $\Omega$ if it is continuous and

$$
\int_{\varphi \neq 0} g_{u}^{p} \mathrm{~d} \nu \leq \int_{\varphi \neq 0} g_{u+\varphi}^{p} \mathrm{~d} \nu \text { for all } \varphi \in N_{0}^{1, p}(\Omega) .
$$

This is one of several equivalent definitions in the literature, see Björn [3, Proposition 3.2 and Remark 3.3] (or [5, Proposition 7.9 and Remark 7.10]). In particular, multiplying $\varphi$ by suitable cut-off functions shows that the inequality in (9.4) can equivalently be required for all $\varphi \in N_{0}^{1, p}(\Omega)$ with bounded support.

If $v$ is locally doubling and supports a local $p$-Poincaré inequality then every $u \in N_{\text {loc }}^{1, p}(\Omega)$ satisfying (9.4) can be modified on a set of zero capacity to become continuous, and thus $p$-harmonic, see Kinnunen-Shanmugalingam [33, Theorem 5.2]. Moreover, it follows from [33, Corollary 6.4] that $p$-harmonic functions obey the strong maximum principle, i.e. if $\Omega$ is connected, then they cannot attain their maximum in $\Omega$ without being constant.

Definition 9.6 A metric space $Y$ is locally annularly quasiconvex around a point $x_{0}$ if there exist $\Lambda \geq 2$ and $r_{0}>0$ such that for every $0<r \leq r_{0}$, each pair of points $x, y \in B\left(x_{0}, 2 r\right) \backslash B\left(x_{0}, r\right)$ can be connected within $B\left(x_{0}, \Lambda r\right) \backslash B\left(x_{0}, r / \Lambda\right)$ by a curve of length at most $\Lambda d(x, y)$.

Lemma 9.7 Let $Y$ be a complete metric space equipped with a globally doubling measure $v$ supporting a global p-Poincaré inequality. Assume that a connected open set $\Omega \subset Y$ is locally annularly quasiconvex around $x_{0} \in \Omega$, with parameters $\Lambda$ and $r_{0}<\operatorname{dist}\left(x_{0}, Y \backslash \Omega\right) / 2 \Lambda$. Let $u$ be a $p$-harmonic function in $\Omega \backslash\left\{x_{0}\right\}$. Then for every $0<r \leq r_{0}$,

$$
\underset{B\left(x_{0}, 2 r\right) \backslash\left\{x_{0}\right\}}{\operatorname{osc}} u \leq C\left(\sum_{k=0}^{\infty}\left(\frac{\left(2^{-k} r\right)^{p}}{v\left(B\left(x_{0}, 2^{-k} r\right)\right)}\right)^{1 /(p-1)}\right)^{1-1 / p}\left(\int_{B\left(x_{0}, 2 \Lambda r\right)} g_{u}^{p} \mathrm{~d} v\right)^{1 / p},
$$

where $C$ depends only on $\Lambda$ and the global doubling and Poincaré constants.

Here $\operatorname{dist}\left(x_{0}, \varnothing\right)$ is considered to be $\infty$.

Remark 9.8 Under the assumptions of Lemma 9.7 and the additional assumption that $r<\frac{1}{4} \operatorname{diam} Y$, the sum in (9.5) is, by e.g. [5, Proposition 6.16], comparable to

$$
\sum_{k=0}^{\infty} \operatorname{cap}_{p}^{Y}\left(B\left(x_{0}, 2^{-k-1} r\right), B\left(x_{0}, 2^{-k} r\right)\right)^{1 /(1-p)} \leq \operatorname{cap}_{p}^{Y}\left(\left\{x_{0}\right\}, B\left(x_{0}, r\right)\right)^{1 /(1-p)},
$$

where the last inequality follows from Lemma 2.6 in Heinonen-KilpeläinenMartio [28] whose proof applies verbatim also in the metric space setting. Thus if $\operatorname{cap}_{p}^{Y}\left(\left\{x_{0}\right\}, B\left(x_{0}, r\right)\right)$ is positive, then the above sum is finite. 
Proof of Lemma 9.7 We can assume that $r_{0}<\frac{1}{2}$ diam $\Omega$. For $0<\rho \leq r_{0}$, we can find $x, y \in B\left(x_{0}, 2 \rho\right) \backslash B\left(x_{0}, \rho\right)$ so that

$$
|u(x)-u(y)| \geq \frac{1}{2} \underset{B\left(x_{0}, 2 \rho\right) \backslash B\left(x_{0}, \rho\right)}{\operatorname{osc}} u .
$$

Let $\gamma$ be a curve in the annulus $B\left(x_{0}, \Lambda \rho\right) \backslash B\left(x_{0}, \rho / \Lambda\right)$ provided by the annular quasiconvexity. Along this curve, we can find a chain of balls $\left\{B_{j}\right\}_{j=1}^{N}$ of radius $\rho / 4 \lambda \Lambda$, such that $N$ is bounded by a constant depending only on $\Lambda$ and the dilation $\lambda$ from the $p$-Poincaré inequality, and

$$
\begin{array}{ll}
2 \lambda B_{j} \subset B\left(x_{0}, 2 \Lambda \rho\right) \backslash B\left(x_{0}, \rho / 2 \Lambda\right) & \text { for } j=1, \ldots, N \\
B_{j} \cap B_{j+1} \neq \varnothing & \text { for } j=1, \ldots, N-1 .
\end{array}
$$

Using Lemma 4.1 in Björn-Björn-Shanmugalingam [12], we thus get that

$$
|u(x)-u(y)| \leq \sum_{j=1}^{N} \underset{B_{j}}{\operatorname{osc}} u \lesssim \frac{\rho}{4 \lambda \Lambda} \sum_{j=1}^{N} \frac{1}{v\left(B_{j}\right)^{1 / p}}\left(\int_{2 \lambda B_{j}} g_{u}^{p} \mathrm{~d} v\right)^{1 / p} .
$$

Since $v$ is globally doubling, we have $v\left(B_{j}\right) \simeq v\left(B\left(x_{0}, \rho\right)\right)$ and so by (9.6) and the uniform bound on $N$,

$$
\underset{B\left(x_{0}, 2 \rho\right) \backslash B\left(x_{0}, \rho\right)}{\operatorname{osc}} u \lesssim \frac{\rho}{\nu\left(B\left(x_{0}, \rho\right)\right)^{1 / p}}\left(\int_{B\left(x_{0}, 2 \Lambda \rho\right) \backslash B\left(x_{0}, \rho / 2 \Lambda\right)} g_{u}^{p} \mathrm{~d} \nu\right)^{1 / p}
$$

Hölder's inequality, together with the last estimate applied to $\rho=r_{k}=2^{-k} r$ then yields

$$
\begin{aligned}
\underset{B\left(x_{0}, 2 r\right) \backslash\left\{x_{0}\right\}}{\mathrm{osc}} u & \leq \sum_{k=0}^{\infty} \underset{B\left(x_{0}, 2 r_{k}\right) \backslash B\left(x_{0}, r_{k}\right)}{\operatorname{osc}} u \\
& \lesssim\left(\sum_{k=0}^{\infty}\left(\frac{r_{k}}{v\left(B\left(x_{0}, r_{k}\right)\right)^{1 / p}}\right)^{p /(p-1)}\right)^{1-1 / p}\left(\sum_{k=0}^{\infty} \int_{A_{k}} g_{u}^{p} \mathrm{~d} \nu\right)^{1 / p},
\end{aligned}
$$

where $A_{k}=B\left(x_{0}, 2 \Lambda r_{k}\right) \backslash B\left(x_{0}, r_{k} / 2 \Lambda\right)$. These annuli have clearly bounded overlap depending only on $\Lambda$, and so (9.5) follows.

The following lemma will be used when proving Theorem 10.5.

Lemma 9.9 Let $(\Omega, d)$ be an A-uniform space and $a \in \partial \Omega$. Then $\bar{\Omega} \backslash\{a\}$ is locally annularly quasiconvex around $a$ with $\Lambda=4 A$.

Proof Let $r>0$ and assume that $x, y \in \Omega \cap B(a, 2 r) \backslash B\left(a, \frac{1}{2} r\right)$. Let $\gamma$ be an arc length parametrized $A$-uniform curve joining $x$ to $y$. Since $l_{\gamma} \leq A d(x, y) \leq 4 A r$, we 
have $\gamma \subset B(a,(2+2 A) r)$. Now, if $\frac{1}{4} r \leq t \leq l_{\gamma}-\frac{1}{4} r$, then

$$
d(a, \gamma(t)) \geq d_{\Omega}(\gamma(t)) \geq \frac{1}{A} \min \left\{t, l_{\gamma}-t\right\} \geq \frac{r}{4 A} .
$$

Similarly, if $d(x, \gamma(t))<\frac{1}{4} r$ or $d(y, \gamma(t))<\frac{1}{4} r$ then $d(a, \gamma(t))>\frac{1}{4} r$. In both cases it follows that $\gamma \cap B\left(a, \frac{1}{4} r\right)=\varnothing$, and the lemma is proved under the assumption that $x, y \in \Omega$.

Finally, if $x, y \in \bar{\Omega} \cap B(a, 2 r) \backslash B(a, r)$ with $x \neq y$, then we find

$x^{\prime}, y^{\prime} \in \Omega \cap\left(B(a, 2 r) \backslash B\left(a, \frac{1}{2} r\right)\right) \quad$ with $\quad d\left(x, x^{\prime}\right) \leq \frac{d(x, y)}{8 A}$ and $d\left(y, y^{\prime}\right) \leq \frac{d(x, y)}{8 A}$.

By the definition of uniform space, $\Omega$ is $A$-quasiconvex, and hence so is $\bar{\Omega}$. Join $x^{\prime}$ to $y^{\prime}$ by a curve $\gamma$ as in the first part of the proof. Concatenating $\gamma$ with the $A$-quasiconvex curves, joining $x$ to $x^{\prime}$ and $y^{\prime}$ to $y$, gives a suitable curve $\tilde{\gamma}$ with length

$$
l_{\tilde{\gamma}} \leq A d\left(x^{\prime}, y^{\prime}\right)+2 \cdot \frac{1}{8} d(x, y) \leq(A+1) d(x, y),
$$

which concludes the proof.

\section{$10 p$-Harmonic Functions on $X$ and $X_{\varepsilon}$}

In this section, we assume that $X$ is a locally compact roughly starlike Gromov $\delta$ hyperbolic space equipped with a complete Borel measure $\mu$ such that $0<\mu(B)<\infty$ for all balls $B \subset X$. We also fix a point $z_{0} \in X$, let $M$ be the constant in the roughly starlike condition with respect to $z_{0}$, and assume that

$$
0<\varepsilon \leq \varepsilon_{0}(\delta), \quad \beta>0 \text { and } 1 \leq p<\infty .
$$

Finally, we let $X_{\varepsilon}$ be the uniformization of $X$ with uniformization center $z_{0}$. When discussing the uniformization $X_{\varepsilon}$, and in particular $C_{p}^{\bar{X}_{\varepsilon}}$, it will always be assumed to be equipped with $\mu_{\beta}$ for the $\beta$ given above.

In this section we shall see that with suitable choices of $p, \varepsilon$ and $\beta$ satisfying $\beta=p \varepsilon$, each $p$-harmonic function on the unbounded Gromov hyperbolic space $(X, d, \mu)$ transforms into a $p$-harmonic function on the bounded space $\left(X_{\varepsilon}, d_{\varepsilon}, \mu_{\beta}\right)$. This fact will make it possible to characterize, under uniformly local assumptions, when there are no nonconstant $p$-harmonic functions with finite $p$-energy on $X$, i.e. when the finite-energy Liouville theorem holds. A function $u$ has finite p-energy with respect to $(X, d, \mu)$ if $\int_{X} g_{u}^{p} \mathrm{~d} \mu<\infty$.

In the setting of complete metric spaces, equipped with a globally doubling measure supporting a global $p$-Poincaré inequality, it was shown in Björn-Björn-Shanmugalingam [12, Theorem 1.1] that the finite-energy Liouville theorem holds on $X$ whenever 
$X$ is either annularly quasiconvex around a point or

$$
\limsup _{r \rightarrow \infty} \frac{\mu\left(B\left(x_{0}, r\right)\right)}{r^{p}}>0 \text { for some fixed point } x_{0} .
$$

The focus of this section will be to consider the finite-energy Liouville theorem for Gromov hyperbolic spaces under uniformly local assumptions.

Proposition 10.1 Let $\Omega \subset X$ be open and $u: \Omega \rightarrow[-\infty, \infty]$ be measurable. Then the following are true:

(a) With $g_{u}$ and $g_{u, \varepsilon}$ denoting the minimal $p$-weak upper gradients of $u$ with respect to $(d, \mu)$ and $\left(d_{\varepsilon}, \mu_{\beta}\right)$, respectively, we have

$$
g_{u, \varepsilon}(x)=g_{u}(x) e^{\varepsilon d\left(x, z_{0}\right)}
$$

and

$$
\int_{\Omega} g_{u}(x)^{p} \mathrm{~d} \mu(x)=\int_{\Omega} g_{u, \varepsilon}(x)^{p} e^{(\beta-p \varepsilon) d\left(x, z_{0}\right)} \mathrm{d} \mu_{\beta}(x) .
$$

(b) $N_{\text {loc }}^{1, p}(\Omega, d, \mu)=N_{\text {loc }}^{1, p}\left(\Omega, d_{\varepsilon}, \mu_{\beta}\right)$.

(c) If $\Omega$ is bounded, then $N^{1, p}(\Omega, d, \mu)=N^{1, p}\left(\Omega, d_{\varepsilon}, \mu_{\beta}\right)$, as sets and with comparable norms (depending only on $\varepsilon, \beta$, p and $\Omega$ ).

Remark 10.2 At first glance it would seem that the minimal $p$-weak upper gradient $g_{u, \varepsilon}$ of $u$ would also depend on the ambient measure $\mu_{\beta}$, but because of the local nature of minimal weak upper gradients and by the fact that the weight $x \mapsto e^{-\beta d\left(x, z_{0}\right)}$ is locally bounded away from both 0 and $\infty$, it follows that $g_{u, \varepsilon}$ indeed does not depend on the choice of $\beta$, see the proof below.

Proof of Proposition 10.1 Clearly, (b) follows directly from (c). To prove (a) and (c), we conclude from (6.3) that $g_{\varepsilon}(x):=g(x) e^{\varepsilon d\left(x, z_{0}\right)}$ is an upper gradient of $u$ with respect to $d_{\varepsilon}$ if and only if $g$ is an upper gradient of $u$ with respect to $d$. Since $p$-weak upper gradients can be approximated by upper gradients, both in the $L^{p}$-norm and also pointwise almost everywhere with respect to $\mu$ and (equivalently) $\mu_{\beta}$, this identity holds also for $p$-weak upper gradients. In particular, (10.1) and (10.2) hold, which proves part (a).

If $\Omega$ is bounded, we also have that $\mu$ and $\mu_{\beta}$ are comparable on $\Omega$, which implies that

$$
\int_{\Omega}|u|^{p} \mathrm{~d} \mu \simeq \int_{\Omega}|u|^{p} \mathrm{~d} \mu_{\beta}
$$

with comparison constants depending on $\beta$ and $\Omega$. Together with (10.2), this implies that $u \in N^{1, p}(\Omega, d, \mu)$ if and only if $u \in N^{1, p}\left(\Omega, d_{\varepsilon}, \mu_{\beta}\right)$, with comparable norms. 
Remark 10.3 The proof of Proposition 10.1 also shows that even if $\Omega$ is not bounded then for $\beta \geq p \varepsilon$,

$$
\|u\|_{N^{1, p}\left(\Omega, d_{\varepsilon}, \mu_{\beta}\right)} \leq\|u\|_{N^{1, p}(\Omega, d, \mu)}
$$

and thus $N^{1, p}(\Omega, d, \mu) \subset N^{1, p}\left(\Omega, d_{\varepsilon}, \mu_{\beta}\right)$.

Proposition 10.4 Let $\Omega \subset X$ be open. If $p=\beta / \varepsilon>1$, then a function $u: \Omega \rightarrow \mathbf{R}$ is $p$-harmonic in $\Omega$ with respect to $(d, \mu)$ if and only if it is p-harmonic in $\Omega$ with respect to $\left(d_{\varepsilon}, \mu_{\beta}\right)$. Moreover, its p-energy is the same in both cases, i.e.

$$
\int_{\Omega} g_{u}^{p} \mathrm{~d} \mu=\int_{\Omega} g_{u, \varepsilon}^{p} \mathrm{~d} \mu_{\beta}
$$

Proof By Proposition 10.1 (b), $u \in N_{\text {loc }}^{1, p}(\Omega, d, \mu)$ if and only if $u \in N_{\text {loc }}^{1, p}\left(\Omega, d_{\varepsilon}, \mu_{\beta}\right)$. Let $\varphi$ be a function with bounded support in $X$. Then $\varphi \in N_{0}^{1, p}(\Omega, d, \mu)$ if and only if $\varphi \in N_{0}^{1, p}\left(\Omega, d_{\varepsilon}, \mu_{\beta}\right)$. Thus (10.2), together with a similar identity for the minimal $p$-weak upper gradients of $u+\varphi$, shows that

$$
\int_{\varphi \neq 0} g_{u}^{p} \mathrm{~d} \mu \leq \int_{\varphi \neq 0} g_{u+\varphi}^{p} \mathrm{~d} \mu \text { if and only if } \int_{\varphi \neq 0} g_{u, \varepsilon}^{p} \mathrm{~d} \mu_{\beta} \leq \int_{\varphi \neq 0} g_{u+\varphi, \varepsilon}^{p} \mathrm{~d} \mu_{\beta} .
$$

It then follows from the discussion after Definition 9.5 that $u$ is $p$-harmonic with respect to $(d, \mu)$ if and only if it is $p$-harmonic with respect to $\left(d_{\varepsilon}, \mu_{\beta}\right)$. Moreover, (10.3) follows directly from (10.2).

Theorem 10.5 Assume that $\mu$ is doubling and supports a $p$-Poincaré inequality, both properties holding for balls of radii $\leq R_{0}$. Assume that $\beta>\beta_{0}$ and $p=\beta / \varepsilon>1$. Then the following are equivalent:

(a) There exists a nonconstant p-harmonic function on $(X, d, \mu)$ with finite p-energy, i.e. the finite-energy Liouville theorem fails for $X$.

(b) There are two disjoint compact sets $K_{1}, K_{2} \subset \partial_{\varepsilon} X$ with positive $C_{p}^{\bar{X}_{\varepsilon}}$ capacity.

After proving the theorem we will give some illustrating examples. But first, before proving the theorem, we will provide several useful characterizations of the second condition (b). The characterization (e), applied to the restriction of $C_{p}^{\bar{X}_{\varepsilon}}$ to the boundary $\partial_{\varepsilon} X$, will be used in the proof of Theorem 10.5 .

Lemma 10.6 Let $Z$ be a separable metric space, and $\mathrm{Cap}(\cdot)$ ) be a monotone, countably subadditive set-function with values in $[0, \infty)$, defined for all subsets of $Z$. Assume that for each Borel set $E \subset Z$,

$$
\operatorname{Cap}(E)=\sup _{K} \operatorname{Cap}(K)
$$

where the supremum is taken over all compact subsets $K \subset E$. 


\section{Define the support of Cap as}

$$
\text { supp Cap }=\{x \in Z: \operatorname{Cap}(B(x, r))>0 \text { for all } r>0\} \text {. }
$$

Then the following are equivalent:

(a) There are two disjoint compact sets $K_{1}, K_{2} \subset Z$ such that $\operatorname{Cap}\left(K_{1}\right)>0$ and $\operatorname{Cap}\left(K_{2}\right)>0$.

(b) There is a Borel set $E \subset Z$ such that $\operatorname{Cap}(E)>0$ and $\operatorname{Cap}(Z \backslash E)>0$.

(c) There is an open set $G \subset Z$ such that $\operatorname{Cap}(G)>0$ and $\operatorname{Cap}(Z \backslash G)>0$.

(d) The support supp Cap contains at least two points.

(e) Cap is not concentrated to one point, i.e. $\operatorname{Cap}(Z \backslash\{a\})>0$ for each $a \in Z$.

If $Y$ is equipped with a globally doubling measure $v$ supporting a global $p$-Poincaré inequality and $p>1$, then $C_{p}^{Y}$ is a Choquet capacity, by [5, Theorem 6.11], and thus satisfies the assumptions above. Hence the assumptions are also satisfied for any restriction of $C_{p}^{Y}$ to any closed subset of $Y$ as well. Example 6.6 in [5] shows that (10.4) can fail if $p=1$.

The assumption (10.4) is only needed to establish the equivalence of (a) and (b). On the other hand, separability is only used to deduce the identity (10.5) below, which in turn is used to show the equivalence of (b)-(e).

Proof We start by showing that

$$
\operatorname{Cap}(Z \backslash \operatorname{supp} \text { Cap })=0 \text {. }
$$

To this end, for each $x \in Z \backslash \operatorname{supp}$ Cap there is $r_{x}>0$ so that $\operatorname{Cap}\left(B\left(x, r_{x}\right)\right)=0$. As $Z$ is Lindelöf (which for metric spaces is equivalent to separability, see e.g. [5, Proposition 1.5]), we can write $Z \backslash$ supp Cap as a countable union of such balls, each of which has zero capacity. Hence the countable subadditivity shows that (10.5) holds.

Now we are ready to prove the equivalences of (a)-(e).

(b) $\Rightarrow$ (a) By (10.4) there are $K_{1} \subset E$ and $K_{2} \subset Z \backslash E$ such that $\operatorname{Cap}\left(K_{1}\right)>0$ and $\operatorname{Cap}\left(K_{2}\right)>0$.

(a) $\Rightarrow$ (c) $\Rightarrow$ (b) These implications are trivial.

(b) $\Rightarrow$ (e) Let $a \in Z$. If $a \in E$, then $\operatorname{Cap}(Z \backslash\{a\}) \geq \operatorname{Cap}(Z \backslash E)>0$. Similarly, if $a \notin E$, then $\operatorname{Cap}(Z \backslash\{a\}) \geq \operatorname{Cap}(E)>0$.

(e) $\Rightarrow$ (d) As $\operatorname{Cap}(Z \backslash\{a\})>0$ for each $a \in Z$, it follows from (10.5) that supp Cap is nonempty. Let $a \in \operatorname{supp} \operatorname{Cap}$. As again $\operatorname{Cap}(Z \backslash\{a\})>0$, and (10.5) holds, there is $b \in \operatorname{supp}$ Cap $\backslash\{a\}$.

(d) $\Rightarrow$ (c) Let $a, b \in \operatorname{supp~Cap,~} a \neq b$, and then let $G=B\left(a, \frac{1}{2} d(a, b)\right)$. Thus $\operatorname{Cap}(G)>0$ as $a \in \operatorname{supp} C a p$, while $\operatorname{Cap}(Z \backslash G) \geq \operatorname{Cap}\left(B\left(b, \frac{1}{2} d(a, b)\right)\right)>0$ since $b \in$ supp Cap.

Proof of Theorem 10.5 By Theorem 6.2, the uniformized space $\left(X_{\varepsilon}, d_{\varepsilon}, \mu_{\beta}\right)$, as well as its closure $\bar{X}_{\varepsilon}$, supports a global $p$-Poincaré inequality and $\mu_{\beta}$ is globally doubling. Moreover, $\bar{X}_{\varepsilon}$ is complete. It thus follows from [5, Theorem 6.11], that $C_{p}^{\bar{X}_{\varepsilon}}$ is a 
Choquet capacity, and in particular satisfies the assumptions in Lemma 10.6, and so does its restriction to $\partial_{\varepsilon} X$.

(b) $\Rightarrow$ (a) Let $f(x):=\operatorname{dist}_{\varepsilon}\left(x, K_{1}\right)$. Since $\bar{X}_{\varepsilon}$ is bounded, we have $f \in N^{1, p}\left(\bar{X}_{\varepsilon}\right)$ and hence there exists a $p$-harmonic function $u$ in $X_{\varepsilon}$ such that $u-f \in N_{0}^{1, p}\left(X_{\varepsilon}\right)$, see Shanmugalingam [37, Theorem 5.6] (or [5, Theorem 8.28 and Definition 8.31]). The function $u$ is denoted $H_{p} f$ in Björn-Björn-Shanmugalingam [10] (and $H f$ in [5]). By the Kellogg property ([10, Theorem 3.9] or [5, Theorem 10.5]), we have $\lim _{X_{\varepsilon} \ni y \rightarrow x} u(y)=f(x)$ on $\partial_{\varepsilon} X$, except possibly for a set of zero $C_{p}^{\bar{X}_{\varepsilon}}$-capacity. Consequently, as $f=0$ on $K_{1}, f>0$ on $K_{2}$ and both $K_{1}$ and $K_{2}$ have positive $C_{p}^{\bar{X}_{\varepsilon}}$-capacity, $u$ must be nonconstant on $X_{\varepsilon}$.

Proposition 10.4 implies that $u \in N_{\text {loc }}^{1, p}(X, d, \mu)$ is $p$-harmonic in $X$ with respect to $(d, \mu)$ as well, and from (10.2) with $\beta=p \varepsilon$ it follows that

$$
\int_{X} g_{u}^{p} \mathrm{~d} \mu=\int_{X_{\varepsilon}} g_{u, \varepsilon}^{p} \mathrm{~d} \mu_{\beta} \leq\|u\|_{N^{1, p}\left(X_{\varepsilon}, d_{\varepsilon}, \mu_{\beta}\right)}^{p}<\infty .
$$

$\neg$ (b) $\Rightarrow \neg$ (a) By Lemma 10.6, there is $a \in \partial_{\varepsilon} X$ such that $C_{p}^{\bar{X}_{\varepsilon}}\left(\partial_{\varepsilon} X \backslash\{a\}\right)=0$. The capacity of $\{a\}$ can be zero or positive.

Let $u$ be a $p$-harmonic function in $(X, d, \mu)$ with finite $p$-energy. Then $u$ is also $p$-harmonic on $\left(X_{\varepsilon}, d_{\varepsilon}, \mu_{\beta}\right)$ with finite $p$-energy, by Proposition 10.4. Applying the global $p$-Poincaré inequality to the ball $B_{\varepsilon}\left(x_{0}, 2 \operatorname{diam}_{\varepsilon} X\right) \cap X_{\varepsilon}=X_{\varepsilon}$, with an arbitrary $x_{0} \in X$, shows that $u \in N^{1, p}\left(X_{\varepsilon}\right)$, cf. [5, Proposition 4.13(d)].

If $\partial_{\varepsilon} X$ has zero $C_{p}^{\bar{X}_{\varepsilon}}$-capacity then it is removable for $p$-harmonic functions in $N^{1, p}\left(X_{\varepsilon}\right)$, by Theorem 6.2 in Björn [4] (or [5, Theorem 12.2]). Hence, an extension of $u$ is $p$-harmonic on the compact connected set $\bar{X}_{\varepsilon}$ and is thus constant by the strong maximum principle.

Finally, assume that $C_{p}^{\bar{X}_{\varepsilon}}(\{a\})>0$. Then $E:=\partial_{\varepsilon} X \backslash\{a\}$ has zero capacity and is thus removable for $p$-harmonic functions in $N^{1, p}\left(X_{\varepsilon}\right)$, by [4, Theorem 6.2] (or [5, Theorem 12.2]). Since $u \in N^{1, p}\left(X_{\varepsilon}\right)$, it follows that an extension of $u$ is $p$-harmonic in the open set $\bar{X}_{\varepsilon} \backslash\{a\}=X_{\varepsilon} \cup E$. By Lemma 9.9, we know that $X_{\varepsilon} \cup E$ is annularly quasiconvex at $a$. Since $C_{p}^{\bar{X}_{\varepsilon}}(\{a\})>0$, it is also true that $\operatorname{cap}_{p}^{\bar{X}_{\varepsilon}}\left(\{a\}, B_{\varepsilon}(a, \rho)\right)>0$ if $\rho<\frac{1}{4} \operatorname{diam}_{\varepsilon} X_{\varepsilon}$, by e.g. [5, Proposition 6.16]. Moreover, $\bar{X}_{\varepsilon}$ is connected. Thus Lemma 9.7, together with the remark after it, implies that for sufficiently small $r>0$,

$$
\underset{B_{\varepsilon}(a, 2 r) \backslash\{a\}}{\operatorname{osc}} u \lesssim\left(\int_{B_{\varepsilon}(a, 2 \Lambda r)} g_{u, \varepsilon}^{p} \mathrm{~d} \mu_{\beta}\right)^{1 / p} .
$$

Since $g_{u, \varepsilon} \in L^{p}\left(\bar{X}_{\varepsilon}, \mu_{\beta}\right)$, the last integral tends to 0 as $r \rightarrow 0$ and we conclude that $\lim _{\bar{X}_{\varepsilon} \backslash\{a\} \ni y \rightarrow a} u(y)$ exists. In particular, $u$ is bounded on the compact set $\bar{X}_{\varepsilon}$. Finally, the strong maximum principle for $p$-harmonic functions on $\bar{X}_{\varepsilon} \backslash\{a\}$ shows that $u$ must be constant on $\bar{X}_{\varepsilon}$. 
Example 10.7 (Continuation of Example 4.2.) We have $C_{d}=2$, and all choices of $R_{0}$ are acceptable. Hence any $\varepsilon, \beta>0$ are allowed. Fixing $\varepsilon>0$ and $1<p<\infty$ and choosing $\beta=p \varepsilon$, we see that the weight in (4.1) becomes

$$
w(z)=\varepsilon^{-1+\beta / \varepsilon}(1 / \varepsilon-|z|)^{-1+\beta / \varepsilon}=\varepsilon^{p-1}(1 / \varepsilon-|z|)^{p-1} .
$$

By considering the functions

$$
u_{j}(z)= \begin{cases}\min \left\{1, \frac{1}{j} \log \frac{1}{1-\varepsilon|z|}\right\}, & \text { if }|z|<\frac{1}{\varepsilon} \\ 1, & \text { if }|z|=\frac{1}{\varepsilon}\end{cases}
$$

for which $\left\|u_{j}\right\|_{N^{1, p}\left(X_{\varepsilon}, \mu_{\beta}\right)} \rightarrow 0$, as $j \rightarrow \infty$, we see that $C_{p}^{\bar{X}_{\varepsilon}}(\{ \pm 1 / \varepsilon\})=0$.

Note that $\mathbf{R}$ does not admit any nonconstant $p$-harmonic function with finite $p$ energy.

Example 10.8 Consider $X=\mathbf{R} \times[-1,1]$, which is a Gromov hyperbolic space when equipped with the Euclidean metric. We equip $X$ with a weighted measure

$$
\mathrm{d} \mu(x, y)=w(x, y) \mathrm{d} \mathcal{L}^{2}(x, y)
$$

such that $(X, \mu)$ is uniformly locally doubling and supports a uniformly local $p$ Poincaré inequality. Fixing $z_{0}=(0,0)$, the uniformization with $\varepsilon=1$ gives a uniform domain $X_{1}$ such that $\partial_{1} X$ consists of two points.

To understand the potential theory and geometry of $X_{1}$ near these two points, consider $z=(x, y) \in X$ such that $x \gg 1$. Then, with $d_{1}$ denoting the uniformized metric on $X_{1}$, we have

$$
d_{1}\left(z, z_{0}\right) \approx \int_{0}^{x} e^{-t} \mathrm{~d} t=1-e^{-x} \text { and } d_{1}((x,-1),(x, 1)) \approx e^{-x}
$$

Here by $d_{1}\left(z, z_{0}\right) \approx 1-e^{-x}$ we mean that $d_{1}\left(z, z_{0}\right) /\left(1-e^{-x}\right) \rightarrow 1$ as $x \rightarrow \infty$. Thus, near the two boundary points, $X_{1}$ is (biLipschitz equivalent to) the diamond region in $\mathbf{R}^{2}$ with corners $( \pm 1,0)$ and $(0, \pm 1)$. The two boundary points of $X_{1}$ are $z_{ \pm}:=( \pm 1,0)$.

Let $\beta=p>1$ and let $\mu_{\beta}$ be the weighted measure on $X_{1}$, given by Definition 4.1. By Theorem 10.5, $X$ supports a nonconstant $p$-harmonic function with finite $p$-energy if and only if both boundary points $z_{ \pm}$have positive $C_{p}^{\bar{X}_{1}}$-capacity. By Björn-BjörnLehrbäck [9, Proposition 5.3], $C_{p}^{\bar{X}_{1}}\left(\left\{z_{+}\right\}\right)>0$ if and only if

$$
\int_{0}^{r_{0}}\left(\frac{r^{p}}{\mu_{\beta}\left(B_{1}\left(z_{+}, r\right)\right)}\right)^{1 /(p-1)} \frac{\mathrm{d} r}{r}<\infty
$$


for some (all) sufficiently small $r_{0}$, where the balls $B_{1}\left(z_{+}, r\right)$ are with respect to the metric $d_{1}$. By the global doubling property of $\mu_{\beta}$ we see that

$$
\mu_{\beta}\left(B_{1}\left(z_{+}, r\right)\right) \simeq \mu_{\beta}\left(B_{1}\left(z_{+}, r\right) \backslash B_{1}\left(z_{+}, \frac{1}{2} r\right)\right) .
$$

In view of (2.3), each of these annuli is (roughly) the image of a rectangular region with fixed size and at distance approximately $\log (1 / r)$ from the base point $z_{0}$. Letting $Q(t)=[t-1, t+1] \times[-1,1]$, we therefore have

$$
\mu_{\beta}\left(B_{1}\left(z_{+}, r\right)\right) \simeq \int_{Q(\log (1 / r))} e^{-\beta x} w(x, y) \mathrm{d} \mathcal{L}^{2}(x, y) .
$$

Since $e^{-\beta x} \simeq r^{\beta}$ on $Q(\log (1 / r))$, we therefore conclude that $C_{p}^{\bar{X}_{1}}\left(\left\{z_{+}\right\}\right)>0$ if and only if

$$
\int_{0}^{r_{0}}\left(\int_{Q(\log (1 / r))} w \mathrm{~d} \mathcal{L}^{2}\right)^{1 /(1-p)} \frac{\mathrm{d} r}{r}<\infty
$$

or equivalently,

$$
\int_{0}^{\infty}\left(\int_{Q(t)} w \mathrm{~d} \mathcal{L}^{2}\right)^{1 /(1-p)} \mathrm{d} t<\infty
$$

An analogous condition holds for $z_{-}$.

Note that when $w \equiv 1$, both (10.6) and its analogue for $z_{-}$fail, showing that the unweighted strip $\mathbf{R} \times[-1,1]$ satisfies the finite-energy Liouville theorem. This special case was obtained in Björn-Björn-Shanmugalingam [12] by a more direct method, without the use of uniformization.

Remark 10.9 The weighted Euclidean real line $(\mathbf{R}, \mu)$, where $\mathrm{d} \mu=w \mathrm{~d} x$ is uniformly locally doubling and supports a uniformly local $p$-Poincaré inequality, can be treated similarly and we obtain that $(\mathbf{R}, \mu)$ supports nonconstant $p$-harmonic functions with finite energy if and only if a condition similar to (10.6) holds on it:

$$
\int_{0}^{\infty}\left(\int_{t-1}^{t+1} w(x) \mathrm{d} x\right)^{1 /(1-p)} \mathrm{d} t<\infty
$$

In [12], this question was studied by different methods and under local assumptions on $w$. It follows from the results in Björn-Björn-Shanmugalingam [11] on local $A_{p}$ weights, that the condition

$$
\int_{0}^{\infty} w(x)^{1 /(1-p)} \mathrm{d} x<\infty
$$

obtained in [12], is equivalent to (10.7) under the local assumptions on $w$. 
We end the paper with the following result which is a direct consequence of Theorem 6.2 together with Lemmas 4.10 and 9.4. In combination with Theorem 10.5, it provides a sufficient condition for the existence of nonconstant $p$-harmonic functions on $(X, d, \mu)$ with finite $p$-energy. Note that the Hausdorff dimension depends only on $\varepsilon, C_{d}$ and $R_{0}$, but not on $\beta$ or $p$.

Proposition 10.10 Assume that $\mu$ is doubling and supports a p-Poincaré inequality, both properties holding for balls of radii at most $R_{0}$. Let $\beta>\beta_{0}$. Assume that the Borel set $E \subset \partial_{\varepsilon} X$ has positive $\kappa$-dimensional Hausdorff measure for some $\kappa>$ $\left(\log C_{d}\right) / \varepsilon R_{0}$. If $p=\beta / \varepsilon \geq 1$, then $C_{p}^{\bar{X}_{\varepsilon}}(E)>0$.

Acknowledgements Open access funding provided by Linköping University. The discussions leading to this paper started in 2013, while the authors were visiting Institut Mittag-Leffler. They continued during the parts of 2017 and 2018 when N. S. was a guest professor at Linköping University, partially funded by the Knut and Alice Wallenberg Foundation, and during the parts of 2019 when A. B. and J. B. were Taft Scholars at the University of Cincinnati. The authors would like to thank these institutions for their kind support and hospitality. A.B. and J.B. were partially supported by the Swedish Research Council grants 2016-03424 and 621-2014-3974, respectively. N.S. was partially supported by the NSF grants DMS-1500440 and DMS1800161. The authors would also like to thank Xining Li for a fruitful discussion on annular quasiconvexity leading to Lemma 9.9.

\section{Compliance with Ethical Standards}

Conflict of interest The authors declare that they have no conflict of interest.

Open Access This article is licensed under a Creative Commons Attribution 4.0 International License, which permits use, sharing, adaptation, distribution and reproduction in any medium or format, as long as you give appropriate credit to the original author(s) and the source, provide a link to the Creative Commons licence, and indicate if changes were made. The images or other third party material in this article are included in the article's Creative Commons licence, unless indicated otherwise in a credit line to the material. If material is not included in the article's Creative Commons licence and your intended use is not permitted by statutory regulation or exceeds the permitted use, you will need to obtain permission directly from the copyright holder. To view a copy of this licence, visit http://creativecommons.org/licenses/by/4.0/.

\section{References}

1. Aikawa, H., Shanmugalingam, N.: Carleson-type estimates for $p$-harmonic functions and the conformal Martin boundary of John domains in metric measure spaces. Michigan Math. J. 53, 165-188 (2005)

2. Bishop, C., Tyson, J.T.: Locally minimal sets for conformal dimension. Ann. Acad. Sci. Fenn. Math. 26, 361-373 (2001)

3. Björn, A.: A weak Kellogg property for quasiminimizers. Comment. Math. Helv. 81, 809-825 (2006)

4. Björn, A.: Removable singularities for bounded $p$-harmonic and quasi(super)harmonic functions on metric spaces. Ann. Acad. Sci. Fenn. Math. 31, 71-95 (2006)

5. Björn, A., Björn, J.: Nonlinear Potential Theory on Metric Spaces. EMS Tracts in Mathematics, vol. 17. European Math. Soc, Zürich (2011)

6. Björn, A., Björn, J.: Local and semilocal Poincaré inequalities on metric spaces. J. Math. Pures Appl. 119, 158-192 (2018)

7. Björn, A., Björn, J.: Poincaré inequalities and Newtonian Sobolev functions on noncomplete metric spaces. J. Differ. Equ. 266, 44-69 (2019)

8. Björn, A., Björn, J., Gill, J., Shanmugalingam, N.: Geometric analysis on Cantor sets and trees. J. Reine Angew. Math. 725, 63-114 (2017)

9. Björn, A., Björn, J., Lehrbäck, J.: Volume growth, capacity estimates, p-parabolicity and sharp integrability properties of $p$-harmonic Green functions (In preparation) 
10. Björn, A., Björn, J., Shanmugalingam, N.: The Dirichlet problem for $p$-harmonic functions on metric spaces. J. Reine Angew. Math. 556, 173-203 (2003)

11. Björn, A., Björn, J., Shanmugalingam, N.: Locally p-admissible measures on $\mathbb{R}$. J. Funct. Anal. 278, $108344(2020)$

12. Björn, A., Björn, J., Shanmugalingam, N.: The Liouville theorem for $p$-harmonic functions and quasiminimizers with finite energy. To appear in Math Z. https://doi.org/10.1007/s00209-020-02536-2

13. Björn, J., Shanmugalingam, N.: Poincaré inequalities, uniform domains and extension properties for Newton-Sobolev functions in metric spaces. J. Math. Anal. Appl. 332, 190-208 (2007)

14. Bonk, M., Heinonen, J., Koskela, P.: Unifomizing Gromov Hyperbolic Spaces. Astérisque, vol. 270 (2001)

15. Bonk, M., Saksman, E.: Sobolev spaces and hyperbolic fillings. J. Reine Angew. Math 737, 161-187 (2018)

16. Bonk, M., Saksman, E., Soto, T.: Triebel-Lizorkin spaces on metric spaces via hyperbolic fillings. Indiana Univ. Math. J. 67, 1625-1663 (2018)

17. Bridson, M., Haefliger, A.: Metric Spaces of Non-positive Curvature. Grundlehren der mathematischen Wissenschaften 319. Springer, Berlin (1999)

18. Buckley, S., Herron, D., Xie, X.: Metric space inversions, quasihyperbolic distance, and uniform spaces. Indiana Univ. Math. J. 57, 837-890 (2008)

19. Buyalo, S., Schroeder, V.: Elements of Asymptotic Geometry. EMS Monographs in Mathematics, vol. 3. European Math. Soc, Zürich (2007)

20. Coornaert, M., Delzant, T., Papadopoulos, A.: Géométrie et théorie des groupes. Lecture Notes in Math, vol. 1441. Springer, Berlin (1990)

21. DiMarco, C.: Fractal curves and rugs of prescribed conformal dimension. Topol. Appl. 248, 117-127 (2018)

22. Gehring, F.W., Osgood, B.G.: Uniform domains and the quasihyperbolic metric. J. Anal. Math. 36, 50-74 (1979)

23. Ghys, É., de la Harpe, P.: Espaces métriques hyperboliques, in Sur les groupes hyperboliques d'après Mikhael Gromov (Bern, 1988). Progress Math. 83, 27-45 (1990)

24. Gromov, M.: Hyperbolic groups, in Essays in Group Theory. Math. Sci. Res. Inst. Publ. 8, 75-263 (1987)

25. Gromov, M.: Metric Structures for Riemannian and Non-Riemannian Spaces. Progress in Math, vol. 152. Birkhäuser, Boston, MA (1999)

26. Hajłasz, P., Koskela, P.: Sobolev met Poincaré. Mem. Am. Math. Soc. 145, 688 (2000)

27. Heinonen, J.: Lectures on Analysis on Metric Spaces. Springer, New York (2001)

28. Heinonen, J., Kilpeläinen, T., Martio, O.: Nonlinear Potential Theory of Degenerate Elliptic Equations, 2nd edn. Dover, Mineola, NY (2006)

29. Heinonen, J., Koskela, P.: Quasiconformal maps in metric spaces with controlled geometry. Acta Math. 181, 1-61 (1998)

30. Heinonen, J., Koskela, P., Shanmugalingam, N., Tyson, J.T.: Sobolev Spaces on Metric Measure Spaces. New Mathematical Monographs, vol. 27. Cambridge Univ. Press, Cambridge (2015)

31. Herron, D., Shanmugalingam, N., Xie, X.: Uniformity from Gromov hyperbolicity. Illinois J. Math. 52, 1065-1109 (2008)

32. Keith, S.: Modulus and the Poincaré inequality on metric measure spaces. Math. Z. 245, 255-292 (2003)

33. Kinnunen, J., Shanmugalingam, N.: Regularity of quasi-minimizers on metric spaces. Manuscr. Math. 105, 401-423 (2001)

34. Koskela, P., MacManus, P.: Quasiconformal mappings and Sobolev spaces. Studia Math. 131, 1-17 (1998)

35. Lehrbäck, J.: Neighbourhood capacities. Ann. Acad. Sci. Fenn. Math. 37, 35-51 (2012)

36. Shanmugalingam, N.: Newtonian spaces: An extension of Sobolev spaces to metric measure spaces. Rev. Mat. Iberoam. 16, 243-279 (2000)

37. Shanmugalingam, N.: Harmonic functions on metric spaces. Illinois J. Math. 45, 1021-1050 (2001)

Publisher's Note Springer Nature remains neutral with regard to jurisdictional claims in published maps and institutional affiliations. 


\section{Affiliations}

\section{Anders Björn ${ }^{1}(\mathbb{D}) \cdot J^{\prime}$ ana Björn ${ }^{1}(\mathbb{D}) \cdot$ Nageswari Shanmugalingam $^{2}$}

$凶 \quad$ Jana Björn

jana.bjorn@liu.se

Anders Björn

anders.bjorn@liu.se

Nageswari Shanmugalingam

shanmun@uc.edu

1 Department of Mathematics, Linköping University, 58183 Linköping, Sweden

2 Department of Mathematical Sciences, University of Cincinnati, P.O. Box 210025, Cincinnati, OH 45221-0025, USA 